\title{
YES kinase controls endothelial junctional plasticity and barrier integrity by regulating VE-cadherin phosphorylation and endocytosis
}

Yi Jin

Uppsala University https://orcid.org/0000-0001-9704-973X

Yindi Ding

Uppsala University

Mark Richards

Uppsala University

Mika Kaakinen

University of Oulu

Anna Szymborska

Max Delbrück Center for Molecular Medicine

Andre Rosa

Max Delbrück Center for Molecular Medicine

\section{Elisabeth Baumann}

Max Delbrück Center for Molecular Medicine

\section{Wolfgang Giese}

Max Delbrück Center for Molecular Medicine https://orcid.org/0000-0003-0872-0929

\section{Andreia Pena}

Faculdade de Medicina da Universidade de Lisboa

\section{Emmanuel Nwadozi}

Uppsala University

Maria Jamalpour

Uppsala University

Katrin Holstein

Max Planck Institute for Molecular Biomedicine https://orcid.org/0000-0003-1540-8378

\section{Miguel Sáinz-Jaspeado}

Uppsala University

\section{Dietmar Vestweber}

Max Planck Institute for Molecular Biomedicine

\section{Michael Welsh}

Uppsala University

\section{Miguel Bernabeu}


University of Edinburgh

\section{Claudio Franco}

Faculdade de Medicina da Universidade de Lisboa

\section{Lauri Eklund}

University of Oulu

\section{Holger Gerhardt}

Max Delbrück Center for Molecular Medicine https://orcid.org/0000-0002-3030-0384

\section{Lena Claesson-Welsh ( $\square$ lena.welsh@igp.uu.se)}

Uppsala University

\section{Article}

\section{Keywords:}

Posted Date: January 10th, 2022

DOI: https://doi.org/10.21203/rs.3.rs-1196695/v1

License: (c) (i) This work is licensed under a Creative Commons Attribution 4.0 International License. Read Full License

Version of Record: A version of this preprint was published at Nature Cardiovascular Research on December 7th, 2022. See the published version at https://doi.org/10.1038/s44161-022-00172-z. 


\section{Abstract}

Vascular Endothelial (VE)-cadherin in endothelial adherens junctions is an essential component of the vascular barrier, critical for tissue homeostasis and implicated in progression of diseases such as cancer and eye diseases. Inhibitors of SRC cytoplasmic tyrosine kinase have been applied to suppress tyrosine phosphorylation of VE-cadherin and thereby to prevent excessive leakage, edema and high interstitial pressure. We show that the SRC-related YES tyrosine kinase rather than SRC, is localized at endothelial cell (EC) junctions. EC-specific YES deletion suppresses VE-cadherin phosphorylation, and arrests VEcadherin at EC junctions. This is accompanied by loss of EC collective migration, and exaggerated agonist-induced macromolecular leakage, while extravasation of monocytes is suppressed. Overexpression of Yes causes ectopic VE-cadherin phosphorylation while vascular leakage is unaffected. In contrast, in EC-specific Src-deficient mice, VE-cadherin internalization is maintained and leakage is suppressed. In conclusion, YES-mediated VE-cadherin phosphorylation regulates its constitutive turnover, required for endothelial junction plasticity and vascular integrity.

\section{Main}

Endothelial cell-cell adhesions form an integral part of the vascular barrier, restricting the passage of molecules and cells across the vessel wall ${ }^{1}$. The barrier in most organs is flexible, allowing remodeling of the embryonic and postnatal vascular beds and exchange of small molecules and fluid to maintain adult tissue homeostasis ${ }^{2}$. During acute inflammation and in a range of chronic diseases, the barrier is weakened, leading to extravasation of blood constituents, promoting inflammation and progression of pathologies such as retinopathies and cancer ${ }^{1-3}$. The maintenance of junctions and barrier function relies on junctional complexes of cell-adhesion molecules formed between adjacent endothelial cells (ECs). Tight junctions limit vascular leakage in the central nervous system (CNS) as well as in arteries and postarterial capillaries in peripheral tissues ${ }^{4,5}$. Adherens junctions, formed by vascular endothelial (VE)cadherin and associated catenins, are crucial for regulating venous and capillary vascular permeability, and leukocyte extravasation as well as collective cell migration ${ }^{6-12}$. According to the consensus model, VE-cadherin is rapidly internalized in response to inflammatory cytokines and vascular endothelial growth factor $A$ (VEGFA), allowing transient opening of gaps at EC contacts ${ }^{1}$. Although tyrosine phosphorylation of VE-cadherin has been implicated in regulation of its internalization, the mechanisms in gap formation and vascular leakage have remained poorly understood ${ }^{13,14}$. This is important, as exaggerated vascular leakage and formation of edema is a driver of disease ${ }^{1}$.

Three main tyrosine phosphorylation sites have been identified in VE-cadherin; Y $658, Y 685$ and $Y 731^{15,16,}$ which have been implicated in distinct processes. Both Y658 and $\mathrm{Y} 685$ are phosphorylated in veins, but not in arteries, as a consequence of flow-induced activation of SRC cytoplasmic tyrosine kinases ${ }^{17}$. Phosphorylated ( $p$ )Y658 relieves tension across VE-cadherin through binding of the polarity protein LGN (Leucine-Glycine-Asparagine repeat), which displaces the VE-cadherin-associated p120 catenin $^{18}$. Phosphorylation of Y685 is induced by flow as well as by inflammatory cytokines and VEGFA $16,17,19$. 
Vascular leakage requires phosphorylation on Y685 as inferred from a Y685- to phenylalanine (F) exchange mutant mouse, which shows suppressed extravasation of large molecular weight tracers or

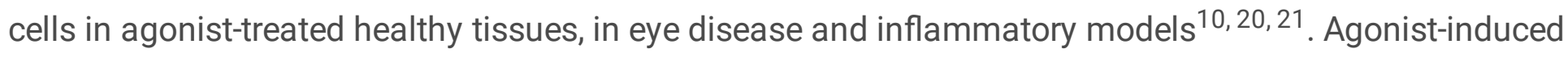
leakage is accompanied by reduced levels of pVE-cadherin, at least in part due to VE-cadherin endocytosis $^{17}$. Y731 is constitutively phosphorylated already upon VE-cadherin synthesis and its dephosphorylation by Src homology phosphatase 2 (SHP2) is a prerequisite for leukocyte extravasation $^{10,22}$. Several additional phosphotyrosine phosphatases (PTPs) such as VE-PTP and density enhanced phosphatase 1 (DEP1; also denoted CD148) have been implicated in regulation of VEcadherin phosphorylation $23,24,25$.

In order to stabilize the vascular barrier in disease, a range of inhibitors have been developed to suppress the activity of SRC, which has been identified as the upstream kinase responsible for the phosphorylation of VE-cadherin on Y658 and Y685 ${ }^{16,17}$. SRC-mediated VE-cadherin phosphorylation on Y685 in response to VEGFA stimulation has been regarded as an important step in VEGFA induced leakage ${ }^{10,16,18 .}$ However, EC-specific Src knockout mice do not display significant changes in VE-cadherin phosphorylation $^{26}$. In addition to SRC, ECs express the highly related cytoplasmic tyrosine kinases YES and FYN. While SRC is implicated in regulation of cell-matrix adhesion ${ }^{26,27}$, the roles played by endothelial YES and FYN have remained poorly understood. In vitro studies have suggested distinct roles for SRC, YES and FYN in regulating EC behaviors, despite their well-conserved SRC Homology 2 (SH2), $\mathrm{SH} 3$ and kinase domains ${ }^{28}$. Accordingly, constitutive global gene inactivation results in abolished VEGFAinduced vascular leakage in constitutive gene inactivated $\mathrm{SrC}^{-/-}$or $\mathrm{Yes}^{-/-}$mice, but not in $\mathrm{Fyn}^{-/-}$mice $^{29}$.

Here, using inducible EC-specific knockout and overexpression mouse models we demonstrate a critical role for YES in the phosphorylation of VE-cadherin at all main phosphorylation sites, Y658, Y685 and Y731. By comparing the effects of losing Src or Yes on VE-cadherin phosphorylation in vivo and in vitro, we conclude that YES is the main kinase that maintains phosphorylation of a subset, about $30 \%$, of the VE-cadherin pool in veins and capillaries, normally leaving most of the pool unphosphorylated. Loss of VE-cadherin phosphorylation suppresses its endocytosis and leads to abnormal barrier properties with blunted collective migration, excessive macromolecular leakage and suppressed leukocyte extravasation. However, forced phosphorylation of VE-cadherin by Yes overexpression does not affect agonist-induced leakage; thus, macromolecular leakage is not controlled solely by the extent of VEcadherin phosphorylation. In contrast, in Src-deficiency, VE-cadherin endocytosis is unaffected and vascular leakage is reduced. Therefore, YES and SRC differently regulate VE-cadherin turnover and adherens junction stability. We conclude that therapeutic efforts to target SRC must consider the potential dire consequence of unintentionally also targeting YES, as the two cytoplasmic kinases have distinct and even opposing roles in the vasculature.

\section{Results}

\section{YES regulates shear stress mediated phosphorylation of VE-cadherin}


Tyrosine phosphorylation of VE-cadherin is high in venules but low in arteries, correlating with the different flow patterns in these vessel types ${ }^{17}$. We investigated the pattern of VE-cadherin phosphorylation (pVE-cadherin) at Y685 in an intact vascular network (whole-mounted developing mouse retina at postnatal day $6 ; \mathrm{P6}$ ), in relation to the relative shear stress level. This was done using computational flow simulation on the PolNet platform ${ }^{30}$ (Figure 1a, b), which models flow based on the vascular plexus geometry and the rheological properties of blood. The specificity of the pY 685 antibody and other pVE-cadherin antibodies used in this study was validated in VE-cadherin mutant mice in which the corresponding tyrosines were mutated to phenylalanine (Supplementary figure 1a-c). The retinal vasculature was segmented into 5 regions: sprouting front (region 1); vein (region 2); capillaries near vein (region 3); capillaries near artery (region 4); artery (region 5) (Figure 1b). Shear stress was the lowest at the sprouting front, correlating to a very low pVE-cadherin signal. In veins and capillaries (regions 2-4), with low to medium shear stress levels, the pVE-cadherin signal was markedly induced. In the artery, with very high shear stress, the pVE-cadherin signal returned to low levels (Figure 1b). In agreement, exposure of human umbilical vein endothelial cells (HUVECs) to low shear stress $\left(3 \mathrm{dyn} / \mathrm{cm}^{2}\right)$ induced accumulation of pVE-cadherin, whereas at high shear stress $\left(20 \mathrm{dyn} / \mathrm{cm}^{2}\right)$, VE-cadherin was unphosphorylated (Figure 1c, d). Furthermore, the appearance of pVE-cadherin in HUVECs correlated with phosphorylation of Src family kinases (SFKs) at Y418, indicative of SFK activity, which was induced under low shear stress and suppressed under high shear stress (Figure 1e-g). A direct and specific relationship between SRC activation and phosphorylation of VE-cadherin has been suggested ${ }^{16}$, however the amino acid sequence around Y418 in the kinase domain of SRC is identical in multiple SFKs. YES, another SFK which is expressed by ECs, was preferentially localized to EC junctions while SRC showed a broad cytoplasmic and nuclear distribution (Figure 1h). Moreover, YES transcript levels were higher than SRC in HUVECs (7.8 \pm 0.5 fold; Figure 1i). Expression of both SRC and YES was further upregulated by low shear stress (Figure 1e). To compare the roles of YES and SRC in regulation of VE-cadherin phosphorylation, YES or SRC silenced HUVECs were subjected to 3 dyn $/ \mathrm{cm}^{2}$ shear stress. The silencing of either YES or SRC did not affect the expression of the other (Supplementary figure 1d, e). However, YES silencing strongly suppressed (82\%) flow-induced pY685 phosphorylation, while SRC silencing resulted in a partial loss (58\%) of pY685 levels (Figure $1 \mathrm{j}, \mathrm{k}$ ).

\section{YES controls in vivo phosphorylation of VE-cadherin on Y658, Y685, and Y731}

To explore the role of YES in vascular function in vivo, a conditional Yes knockout mouse line (Supplementary figure 2a) was crossed with $C d h 5 C r e E R^{T 2}$ mice ${ }^{31}$, to allow tamoxifen-induced EC-specific elimination of Yes expression (denoted Yes iECKO; see Supplementary figure 2b for knockout efficiency). Phosphorylation of VE-cadherin at the different sites, Y658, Y685 and Y731, was investigated by immunostaining. VE-cadherin phosphorylation in Yes iECKO retinas from P6 mice was down-regulated by $74 \pm 15 \%, 78 \pm 10 \%$ and $72 \pm 23 \%$ at $Y 658, Y 685$ and $Y 731$ respectively, compared to littermate controls (Figure 2a-f; see Supplementary figure 1a-c for pY685 and pY731 antibody specificity; the pY658 antibody has been described ${ }^{17}$ ). EC-specific Src knockout (Src iECKO) affected VE-cadherin phosphorylation in P6 retinas to a lesser extent; pVE-cadherin levels decreased by $49 \pm 15 \%$ (Y658), 39 $\pm 6 \%$ (Y685) and $46 \pm 7 \%$ 
(Y731) (Figure 2g-i, Supplementary figure 2c-e). In the Yes iECKO mice, pVE-cadherin levels were dramatically reduced also in large veins such as the vena cava (Supplementary figure $2 \mathrm{f}, \mathrm{g}$ ). Moreover, losing only one allele of Yes in ECs led to about 60\% decrease in pY658 and pY685 VE-cadherin levels in P6 Yes ${ }^{\text {wt/fl: }}$ Cre+ retinas (Supplementary figure $2 \mathrm{~h}, \mathrm{i}$ ). The long-term effect of endothelial Yes deficiency was studied in P22 mice which had been treated with tamoxifen at P1-3. The decrease in VE-cadherin phosphorylation was still evident 3 weeks after the recombination (Supplementary figure 2j, k), which shows that other SFKs did not compensate for Yes-deficiency during this time period.

To study the cell autonomous function of Yes, we introduced the Rosa26R-YFP reporter into the Yes iECKO mouse line. One dose of tamoxifen at P3 generated chimeric recombination, labelling most, but not all, of the Yes-deficient ECs with YFP. In the retinal vasculature of these mice, YFP+ ECs showed $45 \pm 12 \%$ lower pY685-VE-cadherin levels than the adjacent YFP- ECs (Figure 2j, k), indicating that YES controls VE-cadherin phosphorylation in a cell-autonomous manner.

Next, an inducible "floxed-STOP" Yes overexpression mouse model (denoted Yes iECOE) was generated by insertion of the complete mouse Yes cDNA with an upstream floxed transcriptional stop signal into the Hipp11 (H11) locus under the control of the CAG promoter (Supplementary figure 3a). By crossing with the $C d h 5 C r e E R^{T 2}$ mouse and treatment with tamoxifen, EC-specific Yes overexpression was achieved. The overexpressed YES protein localized to endothelial junctions in the retinas of Yes iECOE mice

(Supplementary figure 3b), accompanied by a strong increase in the pY685 VE-cadherin signal. Strikingly, the pY685 VE-cadherin signal was markedly increased at the sprouting front as well as in arteries in Yes iECOE P6 retinas, to a level that was comparable to that in veins and capillaries (Figure 2l, $\mathrm{m}$ ). In mice with chimeric induction of Yes overexpression, ECs displayed a several-fold higher level of VE-cadherin phosphorylation even in capillaries and veins where pVE-cadherin was already high in the control (Figure $2 n, 0)$. This indicates that in WT mice, the extent of VE-cadherin phosphorylation relative to the total VEcadherin pool is limited. Thus, assuming that VE-cadherin in the Yes iECOE mouse is fully phosphorylated, less than $35 \%$ of total VE-cadherin pool was phosphorylated in veins and capillaries in the WT. Therefore we conclude that the limiting factor in flow-regulated VE-cadherin phosphorylation is the expression levels of YES. Interestingly, in the chimeric Yes iECOE mouse retina, branch points on both veins and arteries were more likely to be occupied by Yes OE cells (Supplementary figure 3c, d), indicating that ECs with higher levels of pVE-cadherin may be more resilient to disturbed flow shear stress at vessel bifurcations (note WSS changes at vessel branch points in Figure 1a, rightmost panel). Combined, these results suggest that YES is the main kinase regulating VE-cadherin phosphorylation in vivo.

\section{YES-dependent VE-cadherin phosphorylation is dispensable for retinal vascular development and angiogenic sprouting}

To study the impact of YES-dependent VE-cadherin phosphorylation on vascular development, we compared the developing retinal vasculature in different mouse models with loss- or gain-of-function of YES or VE-cadherin. Endothelial VE-cadherin null mice (Cdh5 iECKO) exhibited a strong hyper-sprouting phenotype (Figure 3a, b). In mice carrying a VE-cadherin mutation at a single phosphosite (Y685F), the 
postnatal retinal vessel development was unaffected (Figure 3c, d). In Yes iECKO mice, where phosphorylation of VE-cadherin was markedly reduced, the vascular development was normal compared to Cre+ control mice $\left(\mathrm{Yes}^{\mathrm{wt} / \mathrm{wt}}\right.$, Cdh5CreER $\left.{ }^{\mathrm{T} 2}+\right)$ (Figure 3e, f). In agreement, VEGFA induced angiogenic sprouting from aortic rings from both control and Yes iECKO mice (Supplementary figure 4a, b), but Yes iECKO sprouts were longer (Supplementary figure 4c). In contrast to the intact vascular development in the Yes iECKO retina, the retinal vascular area was significantly reduced in Yes iECOE mice, compared to control (H11-Yes O/O, Cdh5CreER ${ }^{T 2}+$ ) (Figure 3g, h). We conclude that VE-cadherin is strictly required but its phosphorylation is dispensable for normal sprouting angiogenesis. Moreover, excessive phosphorylation of VE-cadherin interferes with vascular development.

\section{VE-cadherin dependent collective cell migration is impaired by Yes deletion}

During vascular development, ECs migrate from veins towards arteries ${ }^{32}$. Furthermore, endothelial cells migrate collectively in a manner dependent on the plasticity of adherens junctions ${ }^{33}$. To explore whether YES-mediated VE-cadherin phosphorylation impacts endothelial vein to artery migration, the localization of Rosa26R-YFP labelled ECs in Yes iECKO retinas was determined after partial recombination induced at P3 (Figure 4a). To localize YFP+ ECs in flat-mounted retinas, arteries and veins were segmented (Supplementary Figure 5a). The distribution of YFP pixel density between veins and arteries was then mapped in a 2D coordinate system where veins were assigned a referential of 0 and arteries of 1 . In this coordinate system, a referential of 0.5 marks a position at an equal distance from both veins and arteries. In control P7 retinas, the YFP+ ECs were equally distributed between veins and arteries, assuming a reference point of 0.45 (Figure 4a, b, d), whereas in Yes iECKO retinas, the highest YFP+ EC density was found in arteries and there was a general shift of YFP signal towards the arterial side (Figure 4a, c, d; note loss of YFP+ ECs in veins in the Yes iECKO retina in Figure 4a, middle panel). The arterial-favored distribution was not present when recombination was induced at P6, 24 hours before retinas were collected (Figure 4d; Supplementary figure 5b-d). Thus, although vascular development was morphologically unaffected, Yes-deficient ECs were privileged to migrate faster from veins to arteries in the Yes iECKO retina. In retinas with chimeric deletion of VE-cadherin, certain arteries were entirely devoid of VE-cadherin expressing ECs (Supplementary figure 5e), in agreement with that that loss of pVEcadherin-dependent cell-cell adhesion promoted migration towards arteries.

To determine if Yes-deficiency affects EC migration in vitro, HUVECs transfected with control or $Y E S$ siRNA were analyzed in a monolayer scratch assay. Individual cells from the first three rows from the migratory front were tracked and grouped by their initial position (Figure 4e; Supplementary video 1). Control ECs in the different rows migrated collectively at a similar speed while by silencing Yes, the cells in the first and second rows separated from neighbouring cells and migrated at a faster speed (Figure 4f). Thereby, YES-silenced cells from the first and second rows migrated further than the control cells (Figure $4 \mathrm{~g})$.

We further investigated whether the lack of VE-cadherin phosphorylation would affect other cellular processes involved in angiogenesis. EC proliferation in developing retinas was assessed by EdU 
incorporation followed by immunostaining of endothelial specific nuclear protein ERG. The fraction of proliferative ECs was not changed in Yes iECKO mice compared to control ( $\mathrm{Yes}^{f / / f l}, \mathrm{Cdh}^{\mathrm{CC}} \mathrm{CreER^{T2 }}$-) (Supplementary figure $5 \mathrm{f}, \mathrm{g}$ ). Moreover, deleting Yes in ECs did not affect the number of apoptotic cells in postnatal retinas, as demonstrated by immunostaining of cleaved caspase3 (Supplementary Figure 5h, i). Combined, these data show that Yes-deficiency impacts collective EC migration in vivo and in vitro while proliferation and apoptosis remain unaffected.

\section{YES-dependent effects on adherens junction morphology}

Endothelial cell adaptation to microenvironmental demands for remodelling, perfusion or permeability, is dependent on dynamic changes at adherens junctions, which appear as linear, jagged or JAIL (junction associated intermittent lamellipodia) junction morphologies ${ }^{34}$. In the P6 retina, VE-cadherin in arterial ECs formed linear junctions while jagged junctions appeared only on veins and capillaries exhibiting phosphorylated VE-cadherin (Supplementary figure 6). Silencing of YES in HUVECs inhibited formation of jagged junctions at low shear stress (Figure 5a, b) while JAILs increased (Figure 5a, c), indicative of disturbed junction remodelling. Similar changes were observed in vivo, in P6 retinas. Thus, the presence of jagged junctions in the veins of Yes iECKO mice was suppressed (Figure $5 d$, e). While JAlLs were challenging to detect in the retina vessels, the veins of the ear dermal vasculature showed an increase in the JAIL area in Yes iECKO mice (Figure 5f, g), indicative of VE-cadherin clustering. Combined the changes in VE-cadherin morphology with reduced jaggedness and increased JAIL formation in vitro and in vivo in Yes-deficiency indicate that YES steers VE-cadherin trafficking.

\section{YES, but not SRC, is required for VE-cadherin internalization}

Next, the dynamics of VE-cadherin movements in the presence and absence of YES was studied by livecell microscopy. GFP-tagged VE-cadherin was transduced by lentivirus into HUVECs followed by siRNA silencing of $Y E S$ and live-cell imaging. In control cells, VE-cadherin was detected in junction-proximal endocytic vesicles, which moved inwards from the junction. The internalized VE-cadherin vesicles disappeared within 2-5 minutes (Figure 6a, upper panels; Supplementary video 2). In YES-silenced HUVECs, VE-cadherin formed stable clusters in JAlL-like formations, with small vesicles being released from these VE-cadherin clusters (Figure 6a, lower panels; Supplementary video 3). Each of the VEcadherin clusters in si YES cells existed for 15-50 minutes before they gradually disappeared, being replaced by new clusters (Figure 6a, Supplementary video 3 ).

VE-cadherin internalization from the cell surface was further followed by employing an antibody feeding assay. Constitutive internalization of VE-cadherin was efficiently inhibited by silencing YES in HUVECs, whereas silencing of SRC left VE-cadherin unaffected (Figure 6b, c). Also, VEGFA-induced VE-cadherin internalization was blocked by YES-silencing (Figure 6d; supplementary figure 7a). VE-cadherin endocytic vesicles enriched in $\mathrm{PVE}$-cadherin were moreover detected in vivo, just below the plasma membrane, in vena cava ECs from control mice. The frequency of endocytotic vesicles was markedly reduced in Yes 
iECKO mice (Figure 6e, f). Combined, these results indicate that phosphorylation of VE-cadherin is strictly required for its constitutive internalization, which is a prerequisite for junctional plasticity.-

p120 catenin has been implicated in regulation of VE-cadherin endocytosis. The degree of p120 catenin complex formation with VE-cadherin was assessed by co-immunoprecipitation between the two, which showed similar levels of complexes in the basal condition and a trend towards increased p120-VEcadherin association in response to VEGFA in both siControl and si YES HUVECS (Supplementary Figure 7b, c). Thus, complex formation with p120 was not affected by loss of VE-cadherin tyrosine phosphorylation.

\section{Structural analyses of Yes-deficient endothelial junctions}

The decreased VE-cadherin internalization in Yes-deficient ECs could potentially impact junction organization, which was addressed by transmission electron microscopy (TEM) analysis on the mouse ear dermis subjected or not to intradermal injection of VEGFA. Endothelial junction length was not affected by Yes deletion or VEGFA treatment (Figure 7a, b). VEGFA treatment however induced widening of junctions with decreased electron dense cortical actin area per junction length, compared to control mice VEGFA (Figure 7c, d), indicating a transient weakening of cell-cell adhesion and increased vascular permeability. Of note, VEGFA administration did not widen the junctions in Yes iECKO mice (Figure 7c). Instead, in Yes iECKO mice, the electron dense area was smaller than control mice at basal condition and it increased upon VEGFA stimulation (Figure 7d), suggesting disturbed junctional dynamics under Yesdeficiency.

In agreement, live imaging of cells labelled with fluorescent actin revealed that actin stress fibers were misaligned and moved towards the periphery in YES-but not in Control-or SRC-silenced HUVECs (Supplementary videos 4-7). Moreover, VEGFA-treatment significantly increased the proportion of cortical actin in YES-silenced HUVECs, but not in SRC-silenced or control cultures (Figure 7e, f), in agreement with the effect of VEGFA in the ultrastructural TEM analyses of Yes iECKO vessels. In the SRC-silenced cultures, there was instead a trend towards increased proportion of stress fibers relative to cortical actin (Figure 7e, f). However, the activities of Rho-GTPases CDC42, Rac1 and RhoA, which regulate the organization of actin cytoskeleton were unaffected by silencing YES or SRC (Supplementary figure 8a-d).

\section{Vascular permeability is increased in Yes iECKO mice}

Phosphorylation of VE-cadherin has been implicated in vascular permeability and by studying individual, constitutively expressed VE-cadherin tyrosine to phenylalanine mutants the different phosphosites have been implicated in various regulatory aspects ${ }^{10,19-21}$. As VE-cadherin is devoid of phosphotyrosine in the Yes iECKO mice, we could now assay the effects of VE-cadherin phosphorylation on the vascular barrier function in the context of the unphosphorylated, wildtype protein. The basal level of vascular permeability over time was tested by tail vein injection of Evans blue followed by a 2-hour circulation period. Higher levels of Evans blue in the interstitial tissue of the ear skin were detected in Yes iECKO mice (Supplementary figure 9a). The blood-brain-barrier integrity, assessed by the leakage of both Evans blue 
and Cadaverine into the cerebral tissue, was unaffected by Yes deficiency (Supplementary figure 9b, c). Thus, the vascular barrier was compromised in the YES-deficient dermis and potentially other tissues, but not in the CNS.

The permeability of developing retinal vessels was studied by cardiac injection of fixable fluorescent tracer (Dextran-TRITC, $10 \mathrm{kDa}$ ) in P6 mice. Leakage of the tracer into the perivascular tissue was observed preferentially at the vascular sprouting front and neither Yes deletion nor overexpression changed the number of leakage sites at the sprouting front (Figure 8a, b). However, an increased frequency of venous leakage was observed in Yes iECKO retinas (8 leakage sites observed in 11 veins from 10 retinas) in comparison to the control retinas (2 leakage sites observed in 14 veins from 9 retinas) (Figure 8a, c). In contrast, leakage from veins was not elevated in Yes iECOE retinas (Figure 8c).

Next, permeability in mature vessels was investigated in adult mice by intravital confocal imaging of dermal vessels. In the wildtype dermis, pVE-cadherin was detected in some but not all venous vessels an also, unexpectedly, in some arterioles. This is in contrast to the pattern observed in the retina, where veins but not arteries display pVE-cadherin signals. The detection of pVE-cadherin in dermal arterioles may reflect flow-patterns that do not conform to the expected high flow-low phosphorylation pattern, but this remains to be determined. Still, VE-cadherin phosphorylation in dermal vessels was markedly reduced in adult Yes iECKO mice (Supplementary figure 9d, e), similar to the findings in the developing retina. To monitor the leakage, a fluorescent tracer was injected into the tail vein and leakage from dermal vessels in the ear was monitored after local injection of VEGFA (Figure 8d, Supplementary video 8, 9). Leakage was initiated at individual sites in venules and capillaries in both control and Yes iECKO mice (Figure 8e, Supplementary video 10,11). The number of leakage sites was increased in both venules and capillaries in Yes iECKO mice compared to the control (Figure 8f, g). The fluorescent intensity profile at leakage sites showed that leakage appeared sooner after VEGFA injection in Yes iECKO mice compared to the control and that leakage was more vigorous in the absence of Yes (Figure 8h). The number of leakage sites was unaffected in Yes iECOE mice compared to wildtype (Figure 8i, j), indicating that leakage is not directly regulated by VE-cadherin tyrosine phosphorylation. In contrast to the increased leakage observed in Yesdeficiency, Src iECKO mice exhibited a reduced number of leakage site after VEGFA injection compared to control (Figure 8k), in agreement with the presence of non-overlapping downstream targets for SRC and YES and that molecules other than VE-cadherin are involved in maintaining vascular barrier.

The Y731 phosphosite in VE-cadherin is required for leukocyte extravasation. Leukocytes populations were analysed by FACS in the fluids collected by peritoneal lavage in mice $24 \mathrm{~h}$ after saline or VEGFA had been injected. In agreement with the phenotype of Y731F mice ${ }^{10}$, monocyte (CD11 b+ LY6G-) extravasation was reduced in VEGFA treated Yes iECKO mice compared to control (Figure 8I).

Combined, these result show that loss of Yes-mediated phosphorylation of VE-cadherin results in dysregulation of endothelial junctions with increased leakage of macromolecules but suppressed extravasation of inflammatory cells. 


\section{Discussion}

Here, we show that endothelial Yes-deficiency leaves VE-cadherin unphosphorylated at all known phosphotyrosine sites under conditions such as intermediate shear stress and agonist treatment that induce phosphorylation in the wildtype context. As a result, VE-cadherin endocytosis is blocked. The resulting junctional inertia interferes with endothelial collective migration during development, and disturbs barrier function. Basal as well as induced leakage of macromolecules is elevated in Yes-deficient ECs and occurs with abnormal kinetics, while leukocyte extravasation is suppressed. In contrast, overexpression of YES, leading to increased phosphorylation of VE-cadherin in all vessel types, does not affect vascular leakage. Therefore, YES-mediated tyrosine phosphorylation is critical for VE-cadherin's constitutive internalization and junctional pliability, but it is not required for formation of junctional gaps and vascular leakage.

Regulation of endothelial junctions has been ascribed to $S R C^{35,36}$. The viral form, v-SRC, localizes to adherens junctions ${ }^{37}$, and gap junction-dependent cell-cell communication is regulated by c-SRC ${ }^{38}$. Although broad screens for SRC kinase substrates reveal hundreds of molecules yet to be functionally characterized $^{39}$, only a handful have become validated over several decades, including p85-Cortactin, p110-AFAP1, p130Cas, p125FAK and p120-catenin ${ }^{27}$. A common denominator for several of these SRC substrates is the involvement in actin-dependent processes, often with consequence for cell-matrix adhesion. In agreement, EC-specific loss of Src expression results in impaired adhesion properties ${ }^{26}$.

Even less is known about YES substrates. YES phosphorylates the tight junction component occludin both in epithelial and endothelial cells ${ }^{40}$, but the functional consequence is unclear. YES has also been implicated in negative regulation of the cell cycle by phosphorylating cyclin dependent kinase $4^{41}$. As inferred from in vitro analyses, YES plays a role in T-lymphocyte immunity by phosphorylating CD $46^{42}$ and Collapsin Response Mediator Protein 2 (CRMP2) ${ }^{43}$. However, using in vitro kinase-based screen to identify substrates for SRC family kinases, no specific substrate for YES was identified ${ }^{44}$. The important role for YES in ECs is suggested by that YES is expressed at considerably higher levels in HUVECs compared to $S R C$, single-cell transcriptomics shows that Yes is more abundant in ECs than in other cell types in a range of mouse tissues (https://tabula-muris.ds.czbiohub.org/https://tabulamuris.ds.czbiohub.org/ $/)^{45}$.

An important conclusion from the results presented here is that the different SFKs must be targeted individually, e.g. in a therapeutic context but also in scientific studies, as they serve different, sometimes opposing purposes. Yes-deficiency resulted in loss of VE-cadherin phosphorylation, suppressed endocytosis, clustering of VE-cadherin and increased macromolecular leakage. In contrast, in Srcdeficiency, VE-cadherin phosphorylation was reduced albeit to a lesser extent, but VE-cadherin internalization remained unaffected and macromolecular leakage was suppressed. Combined, these data challenge the cause-consequence relationship between VE-cadherin internalization and formation of junctional gaps and macromolecular leakage, which has been the prevailing consensus. Specifically, the 


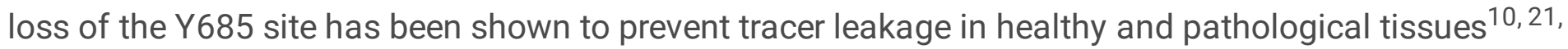
${ }^{46}$. Furthermore, intra-vital studies of mouse dermal vessels show that VEGFA or histamine-induced acute vascular leakage in mature vessels is established at predetermined sites in capillaries and post-capillary venules, where pY685 levels are high ${ }^{5,47}$. In contrast, in the developing retina vasculature, constitutive leakage occurs only at the sprouting front where pY685 level is low. Interestingly, in Yes iECKO mice with non-phosphorylated VE-cadherin, leakage was established also at retina venules. On the other hand, VEGFA-induced leakage was not increased in Yes iECOE mice with the increased pVE-cadherin pool. Combined, these results indicate that VE-cadherin phosphorylation is required for constitutive endocytosis of VE-cadherin and that it sensitizes ECs for rapid VEGFA-induced endocytosis and gap formation at EC junctions. Still, an additional trigger is required for gap formation and macromolecular leakage. Importantly, phosphorylation of VE-cadherin could also be a prerequisite for gap closure. In conclusion, the dysregulated leakage from Yes-deficient vessels may be a consequence of uncoordinated opening and closure of junctional gaps in addition to incomplete sealing in the resting state, due to loss of plasticity, akin to a stiff rubber band that has lost its normal elasticity.

The mechanism underlying the distinct effects of YES and SRC on VE-cadherin may be related to their different subcellular localizations, allowing phosphorylation of different subcellular pools of VE-cadherin, in addition to other kinase-specific substrates. Thus, SRC was broadly distributed in the cytoplasm, while YES accumulated at junctions in HUVEC cultures. SRC is also localized to focal adhesions in freshly seeded ECs where it phosphorylates p125FAK ${ }^{27}$. Endothelial FAK-deficiency results in dismantling of adherens junctions ${ }^{48}$, and VEGFA-induced vascular leakage requires FAK induced phosphorylation of $\beta$ catenin ${ }^{49}$, underscoring the important role of matrix adhesion in regulation of EC junctions. The subcellular localization of cytoplasmic tyrosine kinases is influenced by NH2-terminal fatty acid modification. YES is palmitoylated, while SRC is myristoylated, of consequence for different intracellular trafficking ${ }^{50,51}$. The different subcellular localizations of YES and SRC would also be compatible with their distinct regulation of the actin cytoskeleton as shown here: Yes- but not Src-deficient HUVECs exhibited an increase in cortical actin and loss in actin stress fibers in response to VEGFA. In agreement, VEGFA treatment increased the electron dense, cortical actin-enriched junctional area in Yes-deficient dermal vessels. The abnormal actin arrangements in the absence of Yes, provides a potential mechanistic underpinning for the changes in junctional dynamics (reduced jaggedness and increased JAIL formation) observed in vivo and in vitro, and indeed, the block in VE-cadherin internalization.

Binding of p120-catenin to a VE-cadherin juxtamembrane motif has been implicated in VE-cadherin stabilization ${ }^{13}$, and in agreement, dephosphorylation of VE-cadherin at $Y 658$ increases the binding affinity for p120 in vitro ${ }^{18,52}$. Moreover, the vascular barrier is normal in mice with a mutation of this endocytic motif in VE-cadherin but the vessel density is decreased in the developing retina ${ }^{53}$. In agreement with previous work on phosphorylation-deficient VE-cadherin models ${ }^{17}$, we failed to detect changes in the binding of p120 to VE-cadherin in the absence of YES, in which constitutive VE-cadherin internalization was markedly inhibited. Importantly, in contrast to the VE-cadherin endocytosis deficient 
mutants described previously ${ }^{53}$, the expression levels of both VE-cadherin and p120 were unaffected by Yes-deletion.

The exaggerated macromolecular leakage in $\mathrm{Yes}^{-/}$vessels is at odds with the leakage phenotype of Y685F mice which show suppressed leakage of macromolecules ${ }^{10,21}$ and suppressed extravasation of neutrophils ${ }^{20}$. The difference in phenotypes between the Y685F and the Yes-deficient mouse models may be related to the fact that Yes-deficiency affects additional molecular regulators at endothelial junctions, yet to be discovered. However, the block in monocyte extravasation is in agreement with the phenotype of the $Y 731$ vasculature ${ }^{10}$. Thus, although the phosphosites are similarly regulated by YES, Y658, Y685 and Y731 serve distinct purposes by regulation of extravasation of either macromolecules or inflammatory cells, through different mechanisms and potentially at geographically distinct sites in the vasculature.

Dysregulation of VE-cadherin-mediated adherens junction is manifested in diseases such as endothelialderived tumor Kaposi sarcoma and cerebral cavernous malformation (CCM ${ }^{54,55}$. Moreover, a wide range of diseases are propagated by excess vascular leakage and oedema. The new insights presented here deepens the understanding of the complex regulation of adherens junctions where it is likely that an interplay between several cytoplasmic tyrosine kinases such as YES and SRC orchestrates the process when gaps form and close again and when the vascular surface becomes prepared to dock and expel inflammatory cells. Our results also provide in vivo evidence that specific members of SFKs exert their main functions in different biological processes and therefore, new tools need to be developed to allow individual rather than common targeting of SFKs.

\section{Methods}

Mice. Yes $s^{\text {flox/flox }}$ mouse strain was generated by Taconic/Cyagen by flanking exon 3 with loxP sites, introduced by homologous recombination on the genetic C57BI/ 6 black background (Supplementary

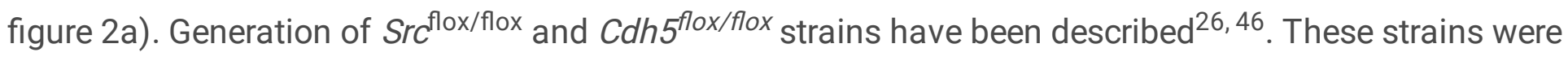
then crossed with Cdh5(PAC)-CreER ${ }^{T 2}$ mice (kind gift from Dr. Ralf Adams, Max-Planck Institute Münster) to generate endothelial specific knockout of $\mathrm{Yes}$, Src or Cdh5. For analysis in mosaic knockout fluorescent

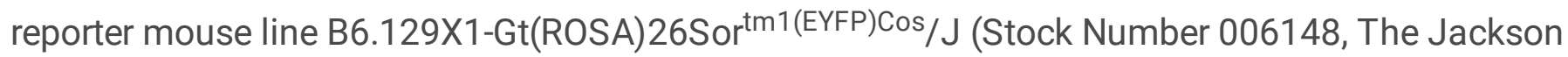
Laboratory) was introduced to indicate Cre activity with YFP expression. The conditional Yes knock-in mouse H11-CAG-STOP-Yes was generated by Taconic/Cyagen using CRISPR/CAS (Supplementary figure 3a). The VE-Cad Y685F mouse has been described ${ }^{10}$. Both males and females are included in the experiments. Animal husbandry and procedures were in accordance with institutional guidelines and approved by the Institutional Review Board for animal experimentation at Uppsala University (permit 5.8.18-06789/2018).

Intravital vascular leakage assay. Intravital imaging of the mouse ear dermis with intradermal injection has been described previously ${ }^{5}$. Briefly, following systemic administration of 2000-kDa Dextran by tailvein injection, mice were anaesthetised by intraperitoneal injection of Ketamine-Xylazine $(120 \mathrm{mg} / \mathrm{kg}$ 
Ketamine, $10 \mathrm{mg} / \mathrm{kg}$ Xylazine) to a surgical level and the ear secured to a solid support. Mice were maintained at a body temperature of $37^{\circ} \mathrm{C}$ for the entire experiment, maximum 90 minutes. Time-lapse imaging was performed using single-photon microscopy (Zeiss LSM 710) and a high N.A waterimmersion lens (CF175 apochromat 25xW N.A1.1, Nikon). For intradermal EC stimulation, a volume of approximately $0.1 \mu$ l recombinant mouse VEGFA164 (Peprotech), concentration $100 \mathrm{ng} / \mu \mathrm{l}$, was injected using a sub-micrometer capillary needle. $10 \mathrm{kDa}$ TRITC Dextran was used as a tracer for VEGFA. Leakage sites were identified in time-lapse imaging, following VEGFA injection, as defined sites of concentrated dextran in the extravascular space. The researcher was blinded to the genotype of the mice when performing the analyses.

In vivo wall shear stress analysis. Flow simulation from the images of retinal vasculature shown by CD31 staining was done using the PolNet platform ${ }^{30}$. Briefly, flat mounted retinas were imaged at an area including one artery, one or two veins and the complete capillary network in between by tile scanning confocal microscope (Leica SP8) using 40X objective. 3D reconstruction of the vascular network was done using maximum projection of the vessels illustrated by CD31 immunostaining. A heat map of relative shear stress level was generated based on the flow simulation by assigning inlet at the artery and outlet at the veins. Regional average shear stress levels were obtained and plotted combined with the pVE-Cadherin levels.

Retinal EC distribution analysis. Chimeric recombination was induced at P3 or P6 by i.p. injection of tamoxifen (100 $\mu \mathrm{g} /$ mouse, Sigma). Retinas were taken at P7 and stained for CD31 and YFP and then flat mounted to slides by cutting into 4 leaflets. Images of whole retinas were taken by z-stack tile scanning using 10X objective on a confocal microscope (Leica SP8). Maximum projection images of whole retinas were used for image segmentation, which was performed with resource to ImageJ. Maximum projection of YFP channel threshold was established to distinguish YFP+ cells from the background signal. Outliers with radius between 0.2-1 $\mu \mathrm{m}$ were removed. CD31 channel (after maximum projection) was used to define the outline of the retina, veins and arteries, and the optic nerve as a mask to define a referential system. For computational analysis a bespoken python-based workflow 2D-coordinate system was employed, that can be accessed on github (https://github.com/wgiese/EC_migration_retina). For every pixel in the microscopic image, three numbers were computed (using the mask as referential): (1) distance to the nearest vein ( $d V D),(2)$ distance to the nearest artery (dAD) and (3) radial distance to the optic nerve ( $r$ ). From these measures, the relative distances by $d A V=d V D /(d V D+d A D)$, were obtained. The EC distribution was computed by performing the operation for all YFP positive pixels, which were used as a proxy for EC distribution.

Vessel leakage in postnatal retina. Recombination was induced at P1-3 by i.p. injection of tamoxifen (100 $\mu \mathrm{g} /$ day/mouse, Sigma). At P6, mice were anesthetized and 10 kDa TRITC-Dextran-Lysine (100 $\mu \mathrm{g} / \mathrm{g}$ body weight, TdB Labs) was injected into the left heart ventricle. After 10 minutes of circulation, mice were decapitated and eyes were taken and fixed in 4\% paraformaldehyde (PFA) (Sigma) for 2 hours at room temperature. A needle puncture on the cornea was made to facilitate fast fixation of the retinas. Retinas were stained with CD31 to visualize blood vessels. Images were acquired by z-stack tile scanning 
in a confocal microscope (Leica SP8). A site of leakage was scored positive if dextran was detected in the perivascular region.

Immune cell infiltration assay. Recombination was induced in 8-12 weeks old mice by gavage of tamoxifen ( $2 \mathrm{mg} /$ day/mouse, Sigma) for 5 consecutive days. The mice were allowed rest for 2 days and saline or VEGFA ( $5 \mu \mathrm{g} / \mathrm{kg}$ body weight) was administered through i.p. injection. After $24 \mathrm{~h}$, mice were sacrificed by cervical dislocation and five $\mathrm{mL}$ of phosphate buffered saline (PBS) was subsequently injected intraperitoneally, and the suspension of recruited cells was collected after abdominal palpation. The cells were immune-stained with CD11b (101206, Biolegend) and LY6G (127608, Biolegend) antibodies for FACS analysis of monocytes.

Transmission Electron microscopy. 8-12 weeks old control or Yes iECKO mice were treated with intradermal injection of VEGFA164 (10 ng, Peprotech) in the ear skin. The other ear served as an untreated control. Mice were then anesthetized and perfused first with $10 \mathrm{~mL}$ Hank's balanced salt solution (HBSS) and then $12 \mathrm{~mL}$ cold fixative (1\% glutaraldehyde (Sigma), 4\% PFA in $0.1 \mathrm{M}$ phosphate buffer) through the left ventricle. Ears were cut and placed in the fixative for $30 \mathrm{~min}$ at $4^{\circ} \mathrm{C}$ and then washed 3 times with PBS, dehydrated and embedded in Epon. Imaging was done with Tecnai G2 Spirit $120 \mathrm{kV}$ transmission electron microscope and Quamesa CCD camera.

Aortic ring assay. Thoracic aortas were taken from mice at P7 and cut into $1 \mathrm{~mm}$ rings after removal of the connective tissues. The rings were embedded between two layers of rat tail collagen, type I (ThermoFisher Scientific) and cultivated in Dulbecco's Modified Eagle's Medium (DMEM) (Gibco) supplemented with $10 \%$ foetal bovine serum (FBS) and VEGFA (Peprotech, $30 \mathrm{ng} / \mathrm{ml}$ ). 4-OH-tamoxifen ( $5 \mu \mathrm{g} / \mathrm{mL}$, Sigma) was added to the culture for 48 hours to induce recombination. After 4 days of initial cultivation samples were fixed in 4\% PFA (Sigma) and analysed by microscopy after immunostaining with antibodies.

Proliferation. Proliferation in the developing retinas was assessed by i.p. injection of 5-ethynyl-2'deoxyuridine (EdU, $100 \mu \mathrm{g} /$ mouse, ThermoFisher Scientific) at postnatal day 6 . Mice were sacrificed 2 hours after injection and retinas were removed and fixed in 4\% PFA (Sigma). EdU staining on retinas was done according to the instructions for the Click-iT EdU imaging kit (ThermoFisher Scientific) apart from 3 $\mathrm{h}$ incubation in the reaction mix at room temperature. The samples were co-stained with antibodies to ERG to identify EC nuclei. Images were taken by confocal microscope (Leica SP8) and analyzed by ImageJ.

Isolation of mouse lung ECs. Mice were treated with tamoxifen (100 $\mu \mathrm{g} /$ day/mouse, Sigma) at P1-3. Lungs were taken at P6 and dissociated to get cell suspension using the MACS dissociation kit (Miltenyi Biotec). Isolation of ECs from mouse lung was done using anti-CD31-antibody (BD Pharmingen)conjugated dynabeads (ThermoFisher Scientific). RNA from the isolated ECs was extracted immediately by using RNeasy mini plus kit (QIAGEN). 
Cell culture. HUVECs (PromoCell) were cultured at $37^{\circ} \mathrm{C}$ with $5 \% \mathrm{CO}_{2}$ in endothelial cell growth $\mathrm{MV} 2$ with supplied supplements (PromoCell). Cells were tested for cell morphology and cell-type specific markers using flow cytometric analyses by the vendor. For VEGFA stimulation, confluent cells were starved in MV2 with $0.2 \%$ FBS without supplements for 5 hours. The same medium with VEGFA165 (50 ng/ml, Peprotech) was then added to the cells and incubated at $37^{\circ} \mathrm{C}$ for indicated time periods.

Scratch wound healing assay. HUVECs were seeded at a density of 50,000 cells/well into a 96-well ImageLockTM tissue culture plate (Essen BioScience) and incubated in MV2 medium overnight followed by standardized scratching using WoundMakerTM (Essen BioScience). Cells were washed with PBS and the plate was placed into an IncuCyte ZOOM (Essen BioScience) and scanned every 15 min for $12 \mathrm{~h}$ using a 10x objective during which data were collected using ZOOM software (Essen BioScience). Cellular migration was analyzed using MTrackJ in ImageJ by manually marking the cells at each time frame. Migration speed of one cell was obtained by dividing the migration distance by time.

Confocal live cell imaging. HUVECs were seeded at full confluency on glass-bottom plates (MatTek) and cultured in MV2 medium overnight. Fresh medium with SiR-actin dye (Cytoskeleton) was added to the cells and incubated for two hours. The cell monolayer was scratched using a $2 \mu \mathrm{L}$ pipette tip and changed to fresh medium. The plates were then placed on a Leica SP8 microscope equipped with a humidified $\mathrm{CO}_{2}$ incubator (Leica Microsystem). Time-laps z-stack images were scanned every 1 min for 3 hours.

In vitro flow treatment. HUVECs were seeded at full confluency on $\mu$-slides (Ibidi) coated with fibronectin $(1 \mu \mathrm{g} / \mathrm{mL}$, Sigma) and cultured overnight in degassed MV2 medium. The slides were then connected to the Ibidi pump system to allow degassed medium flowing through at 3 or $20 \mathrm{dyn} / \mathrm{cm}^{2}$ shear stress for 24 $\mathrm{h}$ at $37^{\circ} \mathrm{C}$ in a $\mathrm{CO}_{2}$ incubator. Static control cells were cultured on the slides in the incubator for the same time period. Cells were fixed in 1\% PFA for 10 min for immunofluorescence or lysed in RIPA buffer (ThermoFisher Scientific) for western blots.

VE-cadherin internalization assay. Control or YES silenced confluent HUVECs were incubated with an antibody against the VE-cadherin extracellular domain (Clone BV6, MABT134, Merck Millipore, $10 \mu \mathrm{g} / \mathrm{mL}$ ) at $4^{\circ} \mathrm{C}$ for 1 hour in MV2 basal medium containing $3 \%$ bovine serum albumin (BSA) (Sigma) without supplements. Unbound antibody was removed by rinsing cells in ice-cold MV2 basal medium. Cells were then cultured at $37^{\circ} \mathrm{C}$ for 4 hours in the presence of $150 \mu \mathrm{M}$ chloroquine (Sigma) in MV2 medium with supplements followed by washing for 8 min with PBS containing $25 \mathrm{mM}$ glycine and $3 \%$ BSA with $\mathrm{Ca}^{2+}$, $\mathrm{Mg}^{2+}, \mathrm{pH} 2.7$, to remove the antibody from cell surface. Cells were rinsed with PBS and fixed with $2 \%$ PFA for $10 \mathrm{~min}$ at room temperature. Total VE-cadherin was stained with an antibody generated in another species (AF1002, R\&D Systems). Samples were mounted on the slides with Fluoromount G (Southern Biotech) and analyzed by confocal imaging.

RNA interference. siRNAs targeting human YES or SRC (Sigma) were transfected into HUVECs by using Lipofectamine RNAiMAX (ThermoFisher Scientific). Control cells were transfected with control siRNA 
which does not target any specific gene. siRNAs were mixed with lipofectamine added to the cells at a concentration of $10 \mathrm{nM}$ and incubated overnight before changing to fresh medium. The cells were used for experiments $48 \mathrm{~h}$ after transfection. 80-95\% knockdown efficiency was validated by quantitative-PCR. YES siRNA: SASI_Hs01_00086922; SRC siRNA: SASI_Hs01_00112907; Control siRNA: MISSION siRNA universal negative control (SCI001).

Immunofluorescence. Mice were sacrificed and tissues were immediately removed and fixed in $2 \%$ PFA (eyes and ears were fixed for $1 \mathrm{~h}$, vena cava was fixed for $15 \mathrm{~min}$ ). Samples were incubated in PBS with $0.5 \%$ Triton X-100 and 1\% BSA for 3 hours at room temperature and then with primary antibodies in the same solution overnight at $4^{\circ} \mathrm{C}$ with agitation. After 3 washes at room temperature, samples were incubated with secondary antibodies overnight at $4^{\circ} \mathrm{C}$, washed 3 times and mounted to slides for imaging. For staining of cultured cells, cells were fixed in 1\% PFA for 10 min after treatment. After fixation, the cells were washed 3 times in PBS and permeabilized in PBS with $0.1 \%$ TritionX-100 for 5 minutes, followed by block in $1 \%$ BSA for 30 minutes and incubation with primary antibodies overnight at $4{ }^{\circ} \mathrm{C}$. Cells were washed 3 times in $0.1 \%$ TritonX-100 and incubated with secondary antibodies for 1 hour at room temperature. They were then washed 3 times and mounted to slides for imaging. Specimens were imaged by Leica SP8 (Leica Microsystems) confocal microscopes. Image processing and analysis was done using ImageJ software.

Antibodies against pVE-cadherin $\mathrm{Y} 658$, $\mathrm{Y} 685$ or $\mathrm{Y} 731$ were generated by immunizing rabbit with phosphor-peptides of corresponding regions of mouse VE-cadherin (New England Peptide). The pY658 antibody was a kind gift from Dr Elisabetta Dejana, IFOM, Milano, Italy and Uppsala University, Sweden). The antibodies were purified and pre-cleared by incubation on fixed and permeabilized $C d h 5$ null mouse ECs before use. Commercial antibodies used are: goat anti mouse VE-cadherin (AF1002, R\&D Systems, 1:500); goat anti-mouse CD31 (AF3628, R\&D Systems, 1:500); chicken anti-GFP (ab13970, Abcam, 1:1000); rabbit anti-ERG (ab92513, Abcam, 1:500); mouse anti-YES (610376, BD Biosciences, 1:400); mouse anti-SRC (Clone GD11, Merck Millipore, 1:400); Alexa Fluor 647-Phalloidin (A-22287, ThermoFisher Scientific, 1:100). Secondary antibodies conjugated with Alexa Fluor dyes were obtained from ThermoFisher Scientific or Jackson ImmunoResearch Laboratories.

Quantitative PCR. RNA was extracted and purified using RNeasy Plus kit (Qiagen). RNA concentrations were measured by Nanodrop spectrophotometer (ThermoFisher Scientific) and adjusted to equal concentration, followed by reverse transcription using SuperScript III (ThermoFisher Scientific). Real-time quantitative PCRs were performed on Bio-Rad real-time PCR machine using Sso advanced qPCR reagent (Bio-Rad). Housekeeping gene ribosomal protein L19 (Rp/19) was used as internal control. The comparative Ct method was used to calculate fold differences.

Primers sequences are: mouse Yes: forward: 5'-AGTCCAGCCATAAAATACACACC-3', reverse: 5'TGATGCTCCCTTTGTGGAAGA-3'; mouse Rp/19: forward: 5'-GGTGACCTGGATGAGAAGGA-3', reverse: 5'TTCAGCTTGTGGATGTGCTC-3'; human YES. forward: 5'-CTCAGGGGTAACGCCTTTTGG-3', reverse: 5' CACCACCTGTTAAACCAGCAG-3'; human SRC. forward: 5'-GACAGGCTACATCCCCAGC-3', reverse: 5'- 
CGTCTGGTGATCTTGCCAAAA-3'; human RPL 19: forward: 5'-TCGCCTCTAGTGTCCTCCG-3', reverse: 5'GCGGGCCAAGGTGTTTTTC-3'.

Rho-GTPase activity assay. Control or YES silenced HUVECs were starved at $37^{\circ} \mathrm{C}$ in basal MV2 medium with $0.2 \%$ FBS for 3 hours, and then stimulated with VEGFA164 (50 ng/mL, Peprotech) for 15 minutes. Activities of Rho GTPases Rac1, CDC42 and RhoA were detected using kit from Cytoskeleton. Briefly, cells were lysed and $300 \mu \mathrm{g}$ of freshly prepared total protein were incubated with Rhotekin-RBD (RhoA) or PAKPBD (CDC42, Rac1) beads for 1 hour on a rotator at $4^{\circ} \mathrm{C}$. Beads were washed 5 times and boiled in Laemmli buffer with $\beta$-Mercaptoethanol. The samples were separated on an SDS-PAGE (4-12\% gradient gel) (ThermoFisher Scientific) and western blots were performed to detect Rac1, CDC42 and RhoA respectively with the antibodies supplied in the kit.

Immunoprecipitation. Control or YES silenced HUVECs were starved at $37^{\circ} \mathrm{C}$ in basal MV2 medium with $0.2 \%$ FBS for 3 hours, and then stimulated with VEGFA164 (50 ng/mL, Peprotech) for 15 minutes. Cells were immediately lysed on ice in lysis buffer ( $50 \mathrm{mM}$ Tris-HCl pH 7.5, $5 \mathrm{mM}$ EDTA, $150 \mathrm{mM} \mathrm{NaCl}, 0.5 \%$ NP-40) supplemented with protease and phosphatase inhibitor cocktails (Roche). Lysates with $300 \mu \mathrm{g}$ of total protein were incubated with $1.5 \mu \mathrm{g}$ VE-cadherin antibody (AF1002, R\&D Systems) overnight at $4^{\circ} \mathrm{C}$. Protein G Sepharose beads (Cytiva) was added to the mixture and incubated for 3 hours at $4^{\circ} \mathrm{C}$. Beads were washed 5 times with lysis buffer and proteins were denatured and released from the beads by boiling in $\beta$-Mercaptoethanol-containing Laemmli buffer.

Western blot. Protein samples were separated on a SDS-PAGE (4-12\% gradient gel) (ThermoFisher Scientific), transferred to nitrocellulose membranes (GE Healthcare), and incubated sequentially with primary and appropriate horseradish peroxidase (HRP)-conjugated secondary antibodies. Signals were detected using the ECL reagent kit (Cytiva). Pictures were obtained by BioRad ChemiDocMP imaging system and analyzed using Image Lab software. The following primary antibodies were used: rabbit antiVE-cadherin Y685 (CP1981, ECM Biosciences, 1:1000); goat anti mouse VE-cadherin (AF1002, R\&D Systems, 1:1000); rabbit anti-pSRC (Y418) (44-660G, ThermoFisher Scientific, 1:1000); mouse anti-YES (610376, BD Biosciences, 1:1000); mouse anti-p120-Catenin (610133, BD Biosciences, 1:1000). Secondary antibodies used were: Horse radish peroxidase (HRP)-conjugated anti-rabbit IgG (NA934, Cytiva); HRP-conjugated anti-mouse IgG (NA931, Cytiva); HRP-conjugated anti-goat IgG (P0449, Dako). Secondary antibodies were used at a dilution of 1:7500.

Statistics and Reproducibility. Statistical analysis was performed using GraphPad Prism software. Statistical significance in comparison between two groups was determined by unpaired two-tailed Student's t-test. Two-way ANOVA was used to compare between groups in time course experiments. Variances were similar between the groups compared. Differences were considered significant with a $P<$ 0.05. For animal experiments, no statistical methods were used for predetermine sample size. The experiments were not randomized. The investigators were not blinded to allocation during experiment and outcome assessment. 
Data availability. The data that support the finding of this study are available from the corresponding authors upon reasonable request.

\section{Declarations}

\section{Acknowledgements}

We gratefully acknowledge Elisabetta Dejana (Uppsala University; IFOM Milano) and Fabrizio Osenigo (IFOM, Milano) for the pY658-VE-cadherin antibody. Expert advice by Dr. Sofia Nordling is greatly appreciated. Euro-Biolmaging ERIC and Biocenter Oulu Electron Microscopy core facility supported by University of Oulu and Biocenter Finland are acknowledged for providing infrastructure allowing ultrastructural analyses. This study was supported by the Swedish Research Council (2020-01349), the Knut and Alice Wallenberg foundation (KAW 2020.0057 and KAW 2019.0276), Fondation Leducq Transatlantic Network of Excellence Grant in Neurovascular Disease (17 CVD 03) to LCW, HG, CAF and MB, Fundação para a Ciência e Tecnologia (PTDC/MED-PAT/31639/2017; CEECIND/04251/2017), European Research Council (679368): CAF, The Academy of Finland (LE380986) to LE.

\section{References}

1. Claesson-Welsh, L., Dejana, E. \& McDonald, D.M. Permeability of the Endothelial Barrier: Identifying and Reconciling Controversies. Trends Mol Med 27, 314-331 (2021).

2. Komarova, Y.A., Kruse, K., Mehta, D. \& Malik, A.B. Protein Interactions at Endothelial Junctions and Signaling Mechanisms Regulating Endothelial Permeability. Circ Res 120, 179-206 (2017).

3. Wettschureck, N., Strilic, B. \& Offermanns, S. Passing the Vascular Barrier: Endothelial Signaling Processes Controlling Extravasation. Physiol Rev 99, 1467-1525 (2019).

4. Nitta, T. et al. Size-selective loosening of the blood-brain barrier in claudin-5-deficient mice. J Cell Biol 161, 653-660 (2003).

5. Honkura, N. et al. Intravital imaging-based analysis tools for vessel identification and assessment of concurrent dynamic vascular events. Nat Commun 9, 2746 (2018).

6. Corada, M. et al. Vascular endothelial-cadherin is an important determinant of microvascular integrity in vivo. Proc Natl Acad Sci U S A 96, 9815-9820 (1999).

7. Allingham, M.J., van Buul, J.D. \& Burridge, K. ICAM-1-mediated, Src- and Pyk2-dependent vascular endothelial cadherin tyrosine phosphorylation is required for leukocyte transendothelial migration. $J$ Immuno/ 179, 4053-4064 (2007).

8. Turowski, P. et al. Phosphorylation of vascular endothelial cadherin controls lymphocyte emigration. J Cell Sci 121, 29-37 (2008). 
9. Schulte, D. et al. Stabilizing the VE-cadherin-catenin complex blocks leukocyte extravasation and vascular permeability. EMBO J 30, 4157-4170 (2011).

10. Wessel, F. et al. Leukocyte extravasation and vascular permeability are each controlled in vivo by different tyrosine residues of VE-cadherin. Nat Immuno/ 15, 223-230 (2014).

11. Hayer, A. et al. Engulfed cadherin fingers are polarized junctional structures between collectively migrating endothelial cells. Nat Cell Biol 18, 1311-1323 (2016).

12. Cao, J. et al. Polarized actin and VE-cadherin dynamics regulate junctional remodelling and cell migration during sprouting angiogenesis. Nat Commun 8, 2210 (2017).

13. Nanes, B.A. et al. p120-catenin binding masks an endocytic signal conserved in classical cadherins. J Cell Biol 199, 365-380 (2012).

14. Adam, A.P. Regulation of Endothelial Adherens Junctions by Tyrosine Phosphorylation. Mediators Inflamm 2015, 272858 (2015).

15. Potter, M.D., Barbero, S. \& Cheresh, D.A. Tyrosine phosphorylation of VE-cadherin prevents binding of p120- and beta-catenin and maintains the cellular mesenchymal state. J Biol Chem 280, 31906-31912 (2005).

16. Wallez, Y. et al. Src kinase phosphorylates vascular endothelial-cadherin in response to vascular endothelial growth factor: identification of tyrosine 685 as the unique target site. Oncogene 26, 10671077 (2007).

17. Orsenigo, F. et al. Phosphorylation of VE-cadherin is modulated by haemodynamic forces and contributes to the regulation of vascular permeability in vivo. Nat Commun 3, 1208 (2012).

18. Conway, D.E. et al. VE-Cadherin Phosphorylation Regulates Endothelial Fluid Shear Stress Responses through the Polarity Protein LGN. Curr Biol 27, 2727 (2017).

19. Li, X. et al. VEGFR2 pY949 signalling regulates adherens junction integrity and metastatic spread. Nat Commun 7, 11017 (2016).

20. Owen-Woods, C. et al. Local microvascular leakage promotes trafficking of activated neutrophils to remote organs. J Clin Invest 130, 2301-2318 (2020).

21. Smith, R.O. et al. Vascular permeability in retinopathy is regulated by VEGFR2 Y949 signaling to VE-cadherin. Elife 9 (2020).

22. Arif, N. et al. PECAM-1 supports leukocyte diapedesis by tension-dependent dephosphorylation of VE-cadherin. EMBO J 40, e106113 (2021). 
23. Nawroth, R. et al. VE-PTP and VE-cadherin ectodomains interact to facilitate regulation of phosphorylation and cell contacts. EMBO J 21, 4885-4895 (2002).

24. Grazia Lampugnani, M. et al. Contact inhibition of VEGF-induced proliferation requires vascular endothelial cadherin, beta-catenin, and the phosphatase DEP-1/CD148. J Cell Bio/ 161, 793-804 (2003).

25. Juettner, V.V. et al. VE-PTP stabilizes VE-cadherin junctions and the endothelial barrier via a phosphatase-independent mechanism. J Cell Biol 218, 1725-1742 (2019).

26. Schimmel, L. et al. c-Src controls stability of sprouting blood vessels in the developing retina independently of cell-cell adhesion through focal adhesion assembly. Development 147 (2020).

27. Reynolds, A.B. et al. SRChing for the substrates of Src. Oncogene 33, 4537-4547 (2014).

28. Werdich, X.Q. \& Penn, J.S. Src, Fyn and Yes play differential roles in VEGF-mediated endothelial cell events. Angiogenesis 8, 315-326 (2005).

29. Eliceiri, B.P. et al. Selective requirement for Src kinases during VEGF-induced angiogenesis and vascular permeability. Mol Cell 4, 915-924 (1999).

30. Bernabeu, M.O. et al. PolNet: A Tool to Quantify Network-Level Cell Polarity and Blood Flow in Vascular Remodeling. Biophys J 114, 2052-2058 (2018).

31. Wang, Y. et al. Ephrin-B2 controls VEGF-induced angiogenesis and lymphangiogenesis. Nature 465, 483-486 (2010).

32. Xu, C. et al. Arteries are formed by vein-derived endothelial tip cells. Nat Commun 5, 5758 (2014).

33. Friedl, P. \& Mayor, R. Tuning Collective Cell Migration by Cell-Cell Junction Regulation. Cold Spring Harb Perspect Bio/ 9 (2017).

34. Cao, J. \& Schnittler, H. Putting VE-cadherin into JAlL for junction remodeling. J Cell Sci 132 (2019).

35. Levinson, A.D., Oppermann, H., Levintow, L., Varmus, H.E. \& Bishop, J.M. Evidence that the transforming gene of avian sarcoma virus encodes a protein kinase associated with a phosphoprotein. Cell 15, 561-572 (1978).

36. Erikson, R.L., Collett, M.S., Erikson, E. \& Purchio, A.F. Evidence that the avian sarcoma virus transforming gene product is a cyclic AMP-independent protein kinase. Proc Natl Acad Sci U SA 76, 62606264 (1979).

37. Rohrschneider, L.R. Adhesion plaques of Rous sarcoma virus-transformed cells contain the src gene product. Proc Natl Acad Sci U S A 77, 3514-3518 (1980). 
38. Azarnia, R., Reddy, S., Kmiecik, T.E., Shalloway, D. \& Loewenstein, W.R. The cellular src gene product regulates junctional cell-to-cell communication. Science 239, 398-401 (1988).

39. Ferrando, I.M. et al. Identification of targets of c-Src tyrosine kinase by chemical complementation and phosphoproteomics. Mol Cell Proteomics 11, 355-369 (2012).

40. Steed, E., Rodrigues, N.T., Balda, M.S. \& Matter, K. Identification of MarvelD3 as a tight junctionassociated transmembrane protein of the occludin family. BMC Cell Biol 10, 95 (2009).

41. Martin, N.G., McAndrew, P.C., Eve, P.D. \& Garrett, M.D. Phosphorylation of cyclin dependent kinase 4 on tyrosine 17 is mediated by Src family kinases. FEBS J 275, 3099-3109 (2008).

42. Lee, S.W. et al. CD46 is phosphorylated at tyrosine 354 upon infection of epithelial cells by Neisseria gonorrhoeae. J Cell Biol 156, 951-957 (2002).

43. Varrin-Doyer, M. et al. Phosphorylation of collapsin response mediator protein 2 on Tyr-479 regulates CXCL12-induced T lymphocyte migration. J Biol Chem 284, 13265-13276 (2009).

44. Takeda, H. et al. Comparative analysis of human SRC-family kinase substrate specificity in vitro. $J$ Proteome Res 9, 5982-5993 (2010).

45. Tabula Muris, C. et al. Single-cell transcriptomics of 20 mouse organs creates a Tabula Muris. Nature 562, 367-372 (2018).

46. Frye, M. et al. Interfering with VE-PTP stabilizes endothelial junctions in vivo via Tie-2 in the absence of VE-cadherin. J Exp Med 212, 2267-2287 (2015).

47. Richards, M. et al. Intra-vessel heterogeneity establishes enhanced sites of macromolecular leakage downstream of laminin alpha5. Cell Rep 35, 109268 (2021).

48. Schmidt, T.T. et al. Conditional deletion of FAK in mice endothelium disrupts lung vascular barrier function due to destabilization of RhoA and Rac1 activities. Am J Physiol Lung Cell Mol Physio/ 305, L291-300 (2013).

49. Chen, X.L. et al. VEGF-induced vascular permeability is mediated by FAK. Dev Cel/ 22, 146-157 (2012).

50. Kasahara, K. et al. Rapid trafficking of c-Src, a non-palmitoylated Src-family kinase, between the plasma membrane and late endosomes/lysosomes. Exp Cell Res 313, 2651-2666 (2007).

51. Sato, I. et al. Differential trafficking of Src, Lyn, Yes and Fyn is specified by the state of palmitoylation in the SH4 domain. J Cell Sci 122, 965-975 (2009).

52. Hatanaka, K., Simons, M. \& Murakami, M. Phosphorylation of VE-cadherin controls endothelial phenotypes via p120-catenin coupling and Rac1 activation. Am J Physiol Heart Circ Physio/ 300, H162- 
$172(2011)$.

53. Grimsley-Myers, C.M. et al. VE-cadherin endocytosis controls vascular integrity and patterning during development. J Cell Bio/ 219 (2020).

54. Nanes, B.A. et al. p120-catenin regulates VE-cadherin endocytosis and degradation induced by the Kaposi sarcoma-associated ubiquitin ligase K5. Mol Biol Cell 28, 30-40 (2017).

55. Dejana, E. \& Orsenigo, F. Endothelial adherens junctions at a glance. J Cell Sci 126, 2545-2549 (2013).

Figures 


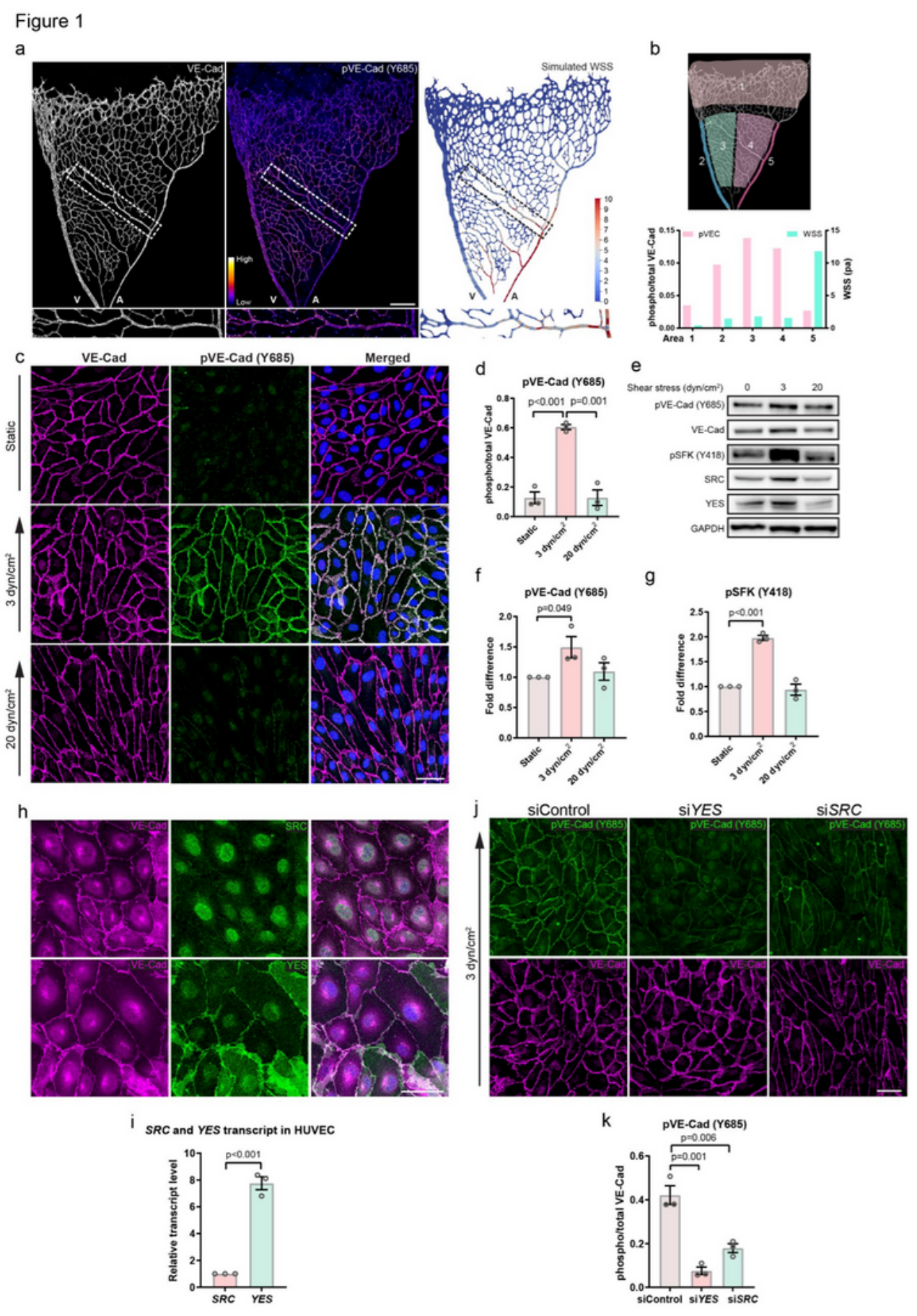

22

Figure 1

Shear stress regulated VE-cadherin phosphorylation is mediated by YES

(a) Whole mount staining of VE-cadherin (left) and pVE-cadherin (Y685) (middle), and computer simulated wall shear stress (WSS; right). An arterial branch (boxed) is shown in the enlarged pictures below. A, artery; V, Vein. Scale bar, $200 \mu \mathrm{m}$. (b) Segmentation of retinas into five regions (upper) and the 
relative regional WSS levels (right Y-axis) plotted (lower) together with the corresponding pVE-cadherin (Y685) levels (left Y-axis) in the different regions. (c) Immunostaining of VE-cadherin (magenta) and pVEcadherin (Y685) (green) in HUVECs cultured in static, $3 \mathrm{dyn} / \mathrm{cm}^{2}$ and $20 \mathrm{dyn} / \mathrm{cm}^{2}$ conditions for $24 \mathrm{~h}$. Scale bar, $50 \mu \mathrm{m}$. (d) Ratio of integrated intensity of pVE-cadherin/total VE-cadherin; $\mathrm{n}=3$ independent experiments. (e) Immunoblots showing pVE-cadherin (Y685), total VE-cadherin, pSFK (Y418), SRC and YES in static or shear stress-treated HUVECs. $(\mathbf{f}, \mathbf{g})$ Ratios of pVE-cadherin/total VE-cadherin and pSFK/GAPDH normalized to static condition, $n=3$ independent experiments. (h) Localization of SRC and YES (green) in HUVECs shown by immunofluorescent staining, VE-cadherin (magenta) is co-stained. (i) Comparison of relative expression level of SRC and YES in HUVECs by qPCR. The comparison was done by generating standard curves for SRC and YES by qPCR from $10^{-2}, 10^{-1}, 1,10,100$ ng RNA samples from HUVECs. $n=3$ independent experiments. (j) Immunofluorescence showing pVE-cadherin (green) and total VE-cadherin (magenta) in HUVECs transfected with control, YES or SRC siRNA followed by exposure to 3 dyn $/ \mathrm{cm}^{2}$ shear stress for $24 \mathrm{~h}$. Scale bar, $50 \mu \mathrm{m}$. (k) Quantification of pVE-cadherin integrated intensity normalized to total VE-cadherin. $n=3$ independent experiments. Bar graphs show mean \pm S.E.M with individual data points. 
Figure 2

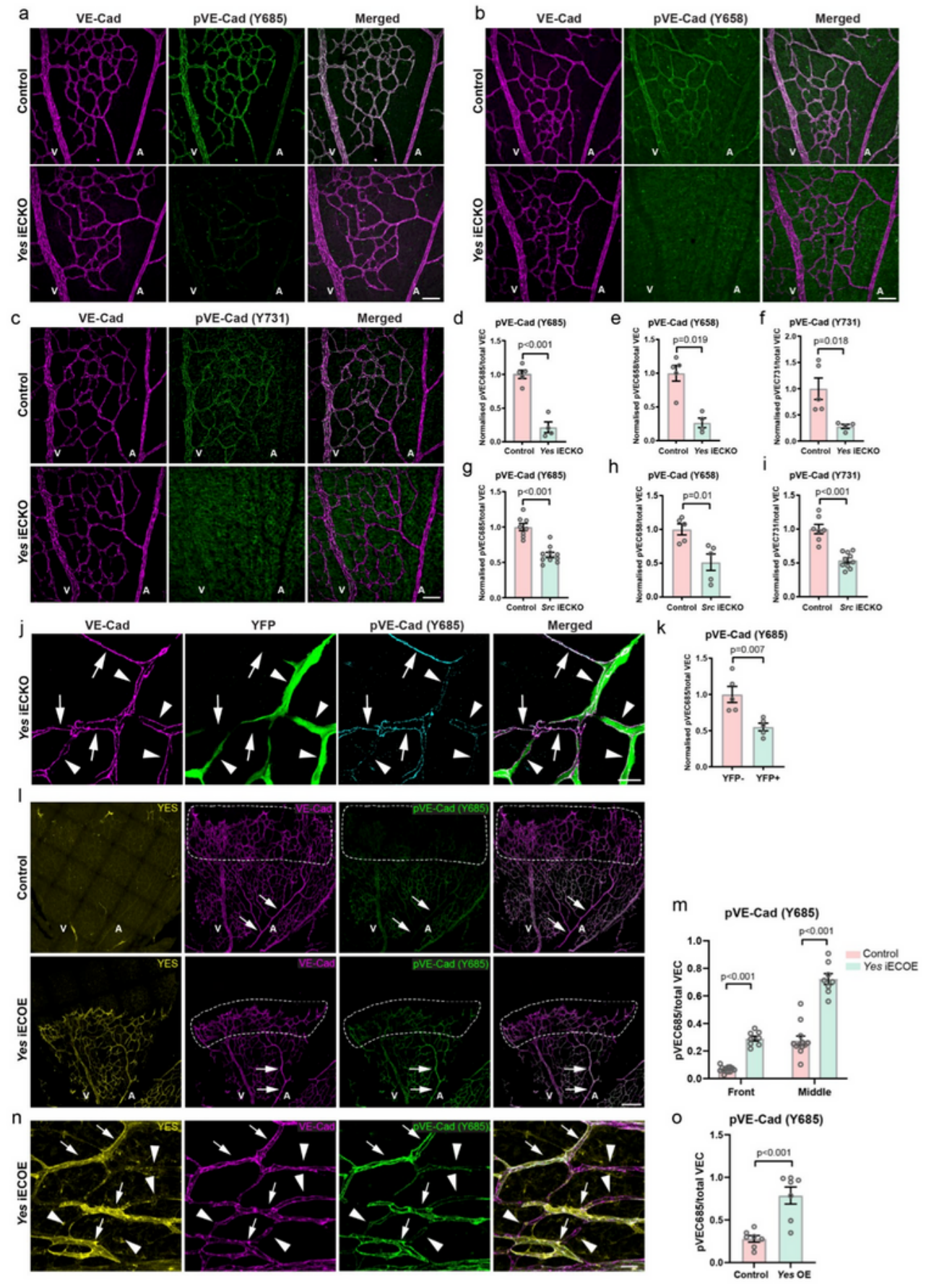

Figure 2

\section{YES mediates phosphorylation of VE-cadherin in vivo}

(a-c) Immunofluorescence of Yes iECKO (Yes fl/fl, Cdh5CreER ${ }^{T 2}+$ ) and control (Yes fl/fl, Cdh5CreER ${ }^{T 2}$ ) P6 retinas showing total VE-cadherin (magenta) and phosphorylated VE-cadherin (green): pY685 (a), pY658 (b) and pY731 (c). Scale bars, 50 mm. (d-f) Quantifications of pVE-cadherin (685) (d), pVE-cadherin (658) 
(e) and pVE-cadherin (731) (f) levels normalized to total VE-cadherin in control and Yes iECKO littermate $\mathrm{P} 6$ retinas. Control, $\mathrm{n}=5$; Yes $\mathrm{iECKO}, \mathrm{n}=4$. ( $\mathbf{g}-\mathbf{i})$ Quantifications of pVE-cadherin in P6 retinas of control (Src $\mathrm{fl} / \mathrm{fl}, \mathrm{Cdh} 5 \mathrm{CreER^{T2 }}{ }^{-}$) and Src iECKO (Src fl/fl, Cdh5CreER ${ }^{T 2}+$ ) mice. (g) pVE-cadherin (685). Control, n=8; Src iECKO, n=10. (h) pVE-cadherin (658). Control, n=5; Src iECKO, n=5. (i) pVE-cadherin (731). Control, n=7; Src iECKO, $n=10$. (j) Immunofluorescence showing correlation between the level of phosphorylated VEcadherin (Y685) and YFP+, Yes-deficient ECs in a P6 retina with chimeric recombination induced by injection of tamoxifen (100 $\mu \mathrm{g} /$ mouse) at P3. Arrows, YFP- ECs; arrowheads, YFP+ ECs. Scale bar, $20 \mu \mathrm{m}$. (k) Quantification of pVE-cadherin (Y685) levels in YFP- and YFP+ ECs. $n=5$ mice. (I) Immunofluorescent images showing YES (yellow), VE-cadherin (magenta) and pVE-cadherin (Y685) (green) in control (upper panel) and an inducible Yes overexpression model (iECOE; lower panel) retinas at P6. Arrows indicate arteries. Dashed lines indicate sprouting front. Scale bar, $200 \mu \mathrm{m}$. $(\mathbf{m})$ quantification of pVE-cadherin (Y685) in the front and middle parts of control and Yes iECOE retinas. Control, $n=12$; Yes iECOE, $n=8$. (n) Immunofluorescence showing phosphorylated VE-cadherin (Y685) in Yes overexpression ECs in a P6 Yes iECOE retina with chimeric recombination. Arrows indicate ECs with Yes overexpression, arrowheads indicate ECs without recombination. Scale bar, $20 \mu \mathrm{m}$. (0) Quantification of pVE-cadherin (Y685) in Yes overexpression ECs and their neighboring non-recombined ECs as shown in (n), $n=7$ mice. Bar graphs show mean \pm S.E.M with individual data points. V, vein; A, Artery. 
Figure 3
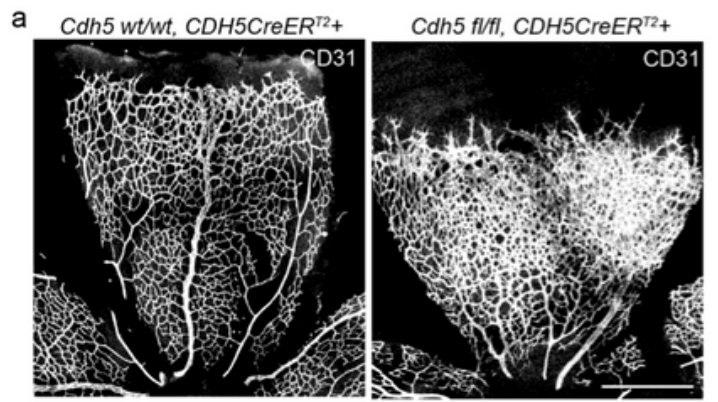

c
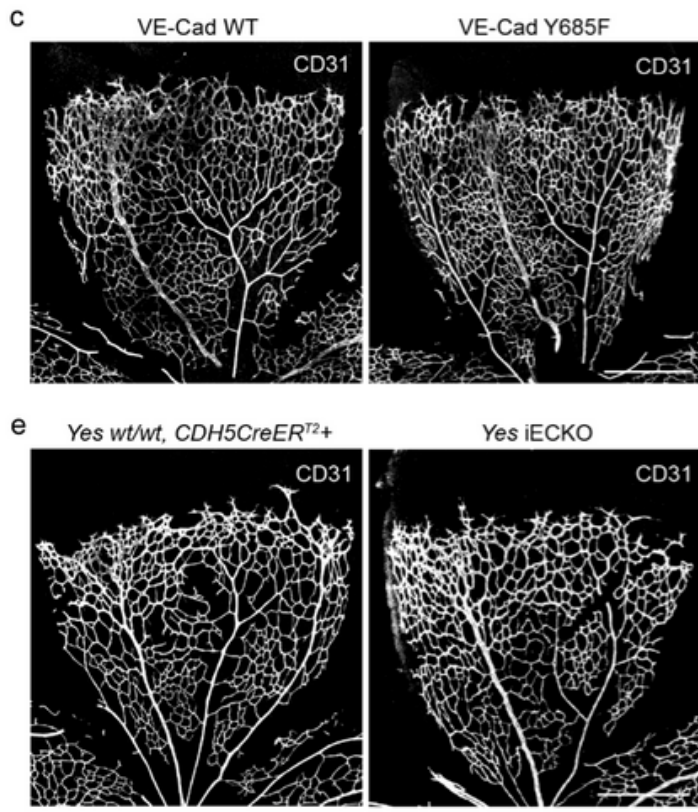

g H11-Yes 0/0, CDH5CreER ${ }^{T 2}+$

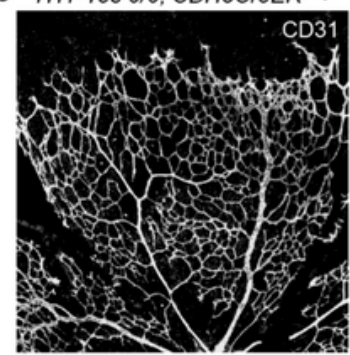

Yes IECKO

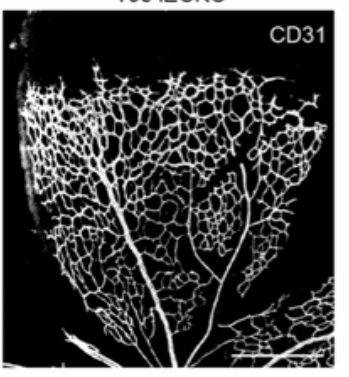

Yes IECOE

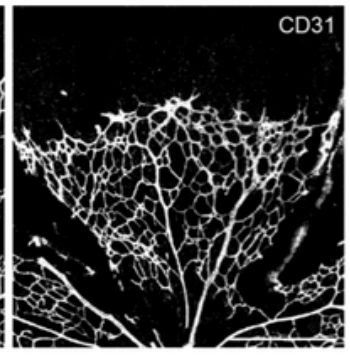

b

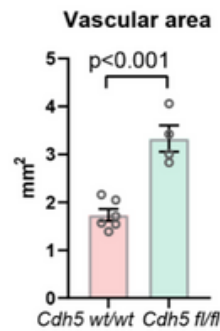

d

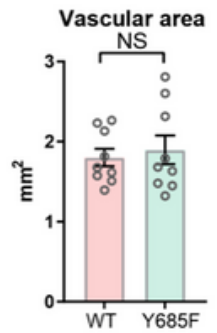

f

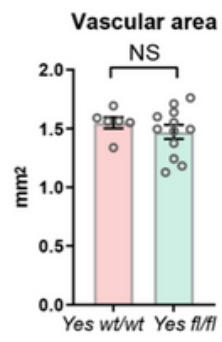

h

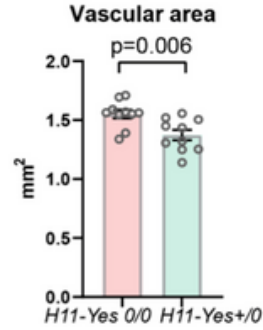

Figure 3

Vascular development in mouse models of VE-cadherin IECKO, VE-cadherin Y685F mutation, Yes iECKO and Yes IECOE

(a) Vasculature in developing retinas of P6 control (Cdh5 wt/wt, Cdh5CreER $\left.{ }^{T 2}+\right)$ and VE-cadherin iECKO (Cdh5 fl/fl, Cdh5CreER ${ }^{T 2}+$ ) mice, shown by CD31 immunostaining. (b) Quantification of vascular area in 
control and VE-cadherin iECKO retinas. Control, $n=6$; Cdh5 iECKO, $n=4$. (c) CD31-positive retinal vasculature in WT and VE-cadherin Y685F mutant mice at P6. Quantification of vascular area in littermates is shown in (d), WT, n=9; Y685F, n=9. (e) Retinal vasculature in Yes $w t / w t, C d h 5 C r e E R^{T 2}+$ and

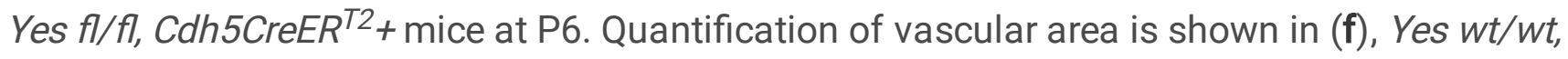
Cdh5CreER ${ }^{T}, \mathrm{n}=6$; Yes fl/fl, Cdh5CreER ${ }^{T 2}+\mathrm{n}=12$. (g) Retinal vasculature in H17-Yes 0/0, Cdh5CreER ${ }^{T 2}+$ and H17-Yes +10 , Cdh5CreER ${ }^{T 2}+$ mice at P6. Quantification of vascular area is shown in (h), H17-Yes 0/O, $\mathrm{n}=10 ; H 11-Y e s+/ 0, \mathrm{n}=10$. Bar graphs show mean \pm S.E.M with individual data points. Scale bars, $500 \mu \mathrm{m}$. 
Figure 4

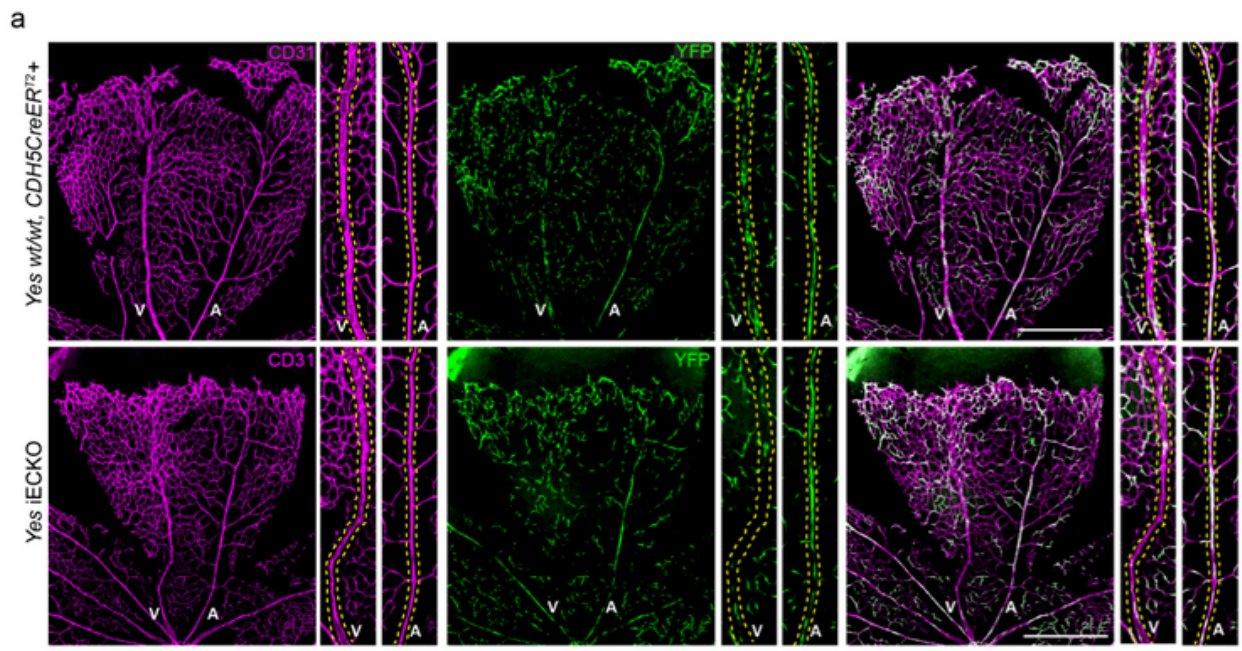

b Distribution of YFP in Control, P7

C Distribution of YFP in Yes IECKO, P7

d
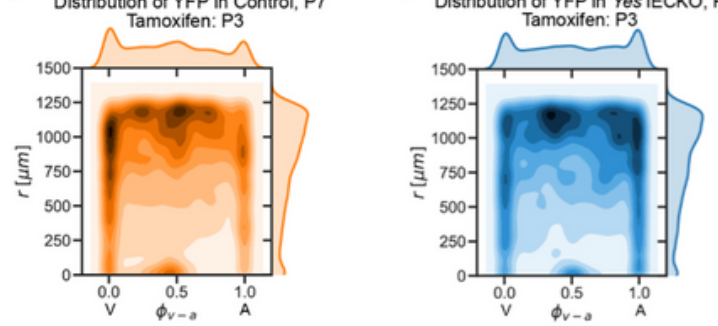

Localization of YFP+ ECs between $\mathrm{V}$ and $\mathrm{A}$ in $\mathrm{P7}$ retinas
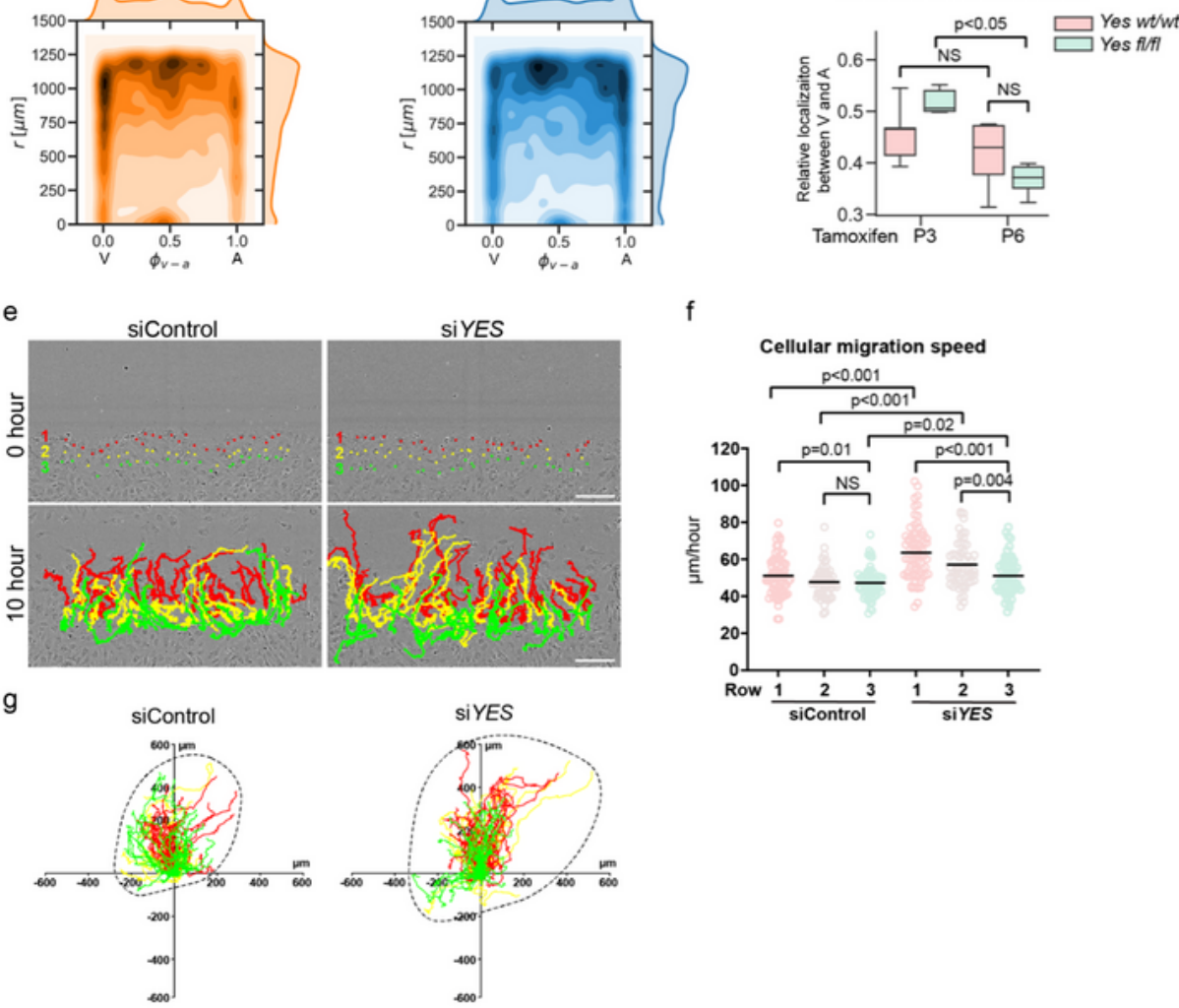

Figure 4

\section{EC localization is affected by VE-cadherin or Yes deletion due to disturbed collective cell migration}

(a) Chd5Cre-induced expression of YFP (green) in $w t / w t, C d h 5 C r e E R^{T 2}+$, and $Y e s f l / f l, C d h 5 C r e E R^{T 2}+$, at P7 after tamoxifen (100 $\mathrm{\mu g} /$ mouse) injection at P3. CD31 (magenta) counter staining was performed to show all ECs. Veins and arteries are shown enlarged to the right with vessel outlines marked by yellow 
dashed lines. V, vein; A, Artery. Scale bars, $500 \mu \mathrm{m}$. (b, c) Localization analysis of YFP+ ECs in P7 retinas after tamoxifen treatment at P3. X-axis indicates the position of veins at 0.0 and arteries at 1.0. Y-axis indicates the measured regional vessel areas up to $1250 \mu \mathrm{m}$ from the optic nerve. Heat map intensity indicates the average density of YFP+ cells analyzed from 6 retinas. (d) Graph showing comparison of relative localization between vein and artery where YFP+ cells are located in P7 retinas treated with tamoxifen at P3 or P6. (e) Individual HUVECs tracked manually during migration in a scratch wound assay. The tracks from cells at different initial positions in rows 1-3 are indicated by colors (first, red; second, yellow; third, green). Scale bars, $100 \mu \mathrm{m}$. (f) Migratory speed of individual cells calculated as length of track $(\mu \mathrm{m}) /$ time (hour). Cells are grouped by their initial positions pooled from 3 independent experiments. siControl, $n=76,59,63$ for cells initially at row 1, 2, 3 respectively; si YES, $n=74,58,61$ for cells initially at row 1, 2, 3 respectively. Graph shows the mean values and individual data points. (g) The migratory tracks of analyzed control or YES silenced HUVECs in (e) are shown in by defining the initial position as $(0,0)$ for all the cells. Dashed lines indicate the maximum travel range of the cells during $10 \mathrm{~h}$. 
Figure 5

a

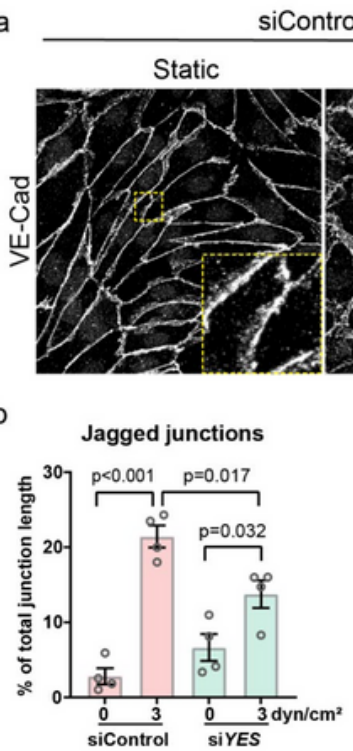

siControl

$3 \mathrm{dyn} / \mathrm{cm}^{2}$

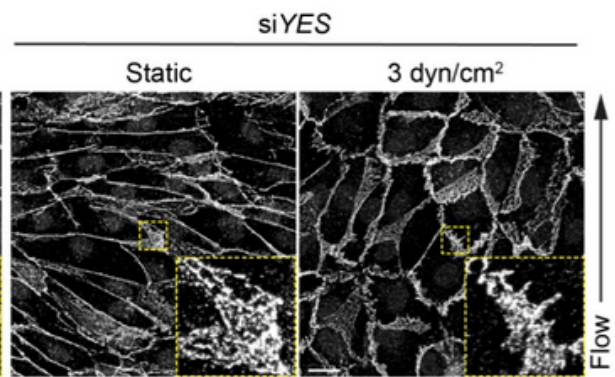

b

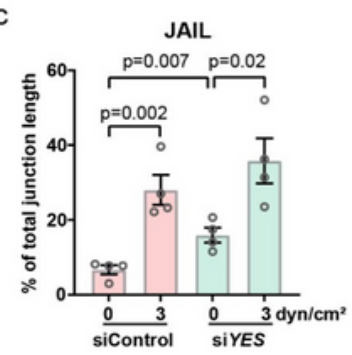

d
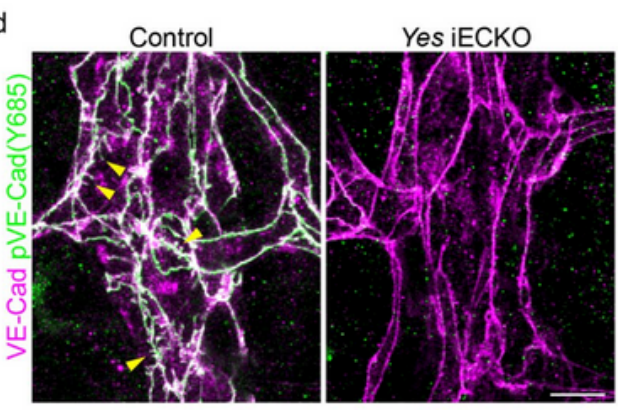

e
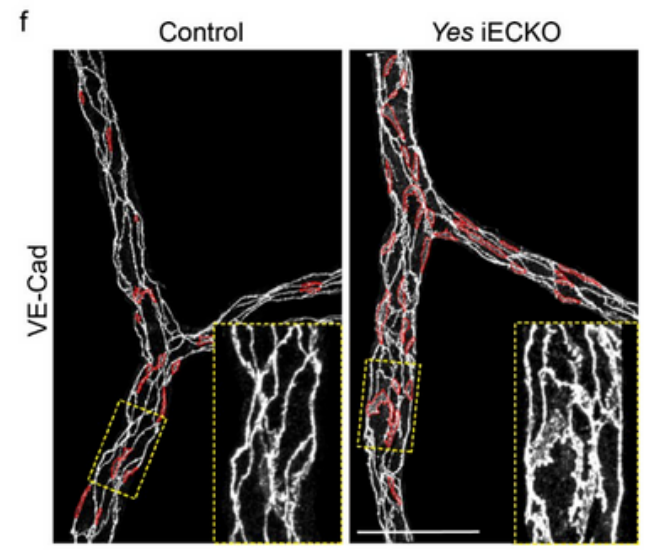

Jagged junctions

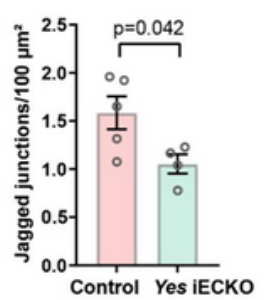

g

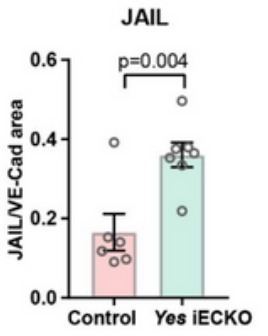

Figure 5

\section{Endothelial junction morphology is altered by Yes deletion}

(a) Immunostaining of VE-cadherin on HUVECs transfected with control or YES siRNA and cultured under static or $3 \mathrm{dyn} / \mathrm{cm}^{2}$ flow conditions. Boxed regions show representatives of altered VE-cadherin morphologies, magnified to the lower right. Scale bar, $20 \mu \mathrm{m}$. Quantifications of jagged and JAIL 
junctions are shown in (b, c), $n=4$ independent experiments. (d) Morphology of adherens junctions in the veins of retinas vessels at P6 illustrated by immunostaining of VE-cadherin (magenta) and pVE-cadherin (Y685) (green). Yellow arrowheads indicate jagged junctions. Scale bar, $10 \mu \mathrm{m}$. (e) Quantification of jagged structures $/ 100 \mu \mathrm{m}^{2}$ VE-cadherin area in control and Yes $\mathrm{iECKO}$ mouse retinas. Control, $\mathrm{n}=5$ mice; Yes iECKO, n=4 mice. (f) Immunostaining of VE-cadherin shows endothelial adherens junctions in adult mice (8-12 weeks) dermal vessels 3 days after tamoxifen induced recombination. JAILs are indicated by red circles. Boxed regions show representatives of altered VE-cadherin morphologies, magnified to the lower right. Scale bar, $50 \mu \mathrm{m}$. (g) Ratios of JAIL area/VE-cadherin area in control and Yes iECKO mice. Control, $n=6$ mice; Yes iECKO, $n=7$ mice. All bar graphs show mean \pm S.E.M with individual data points. 
Figure 6
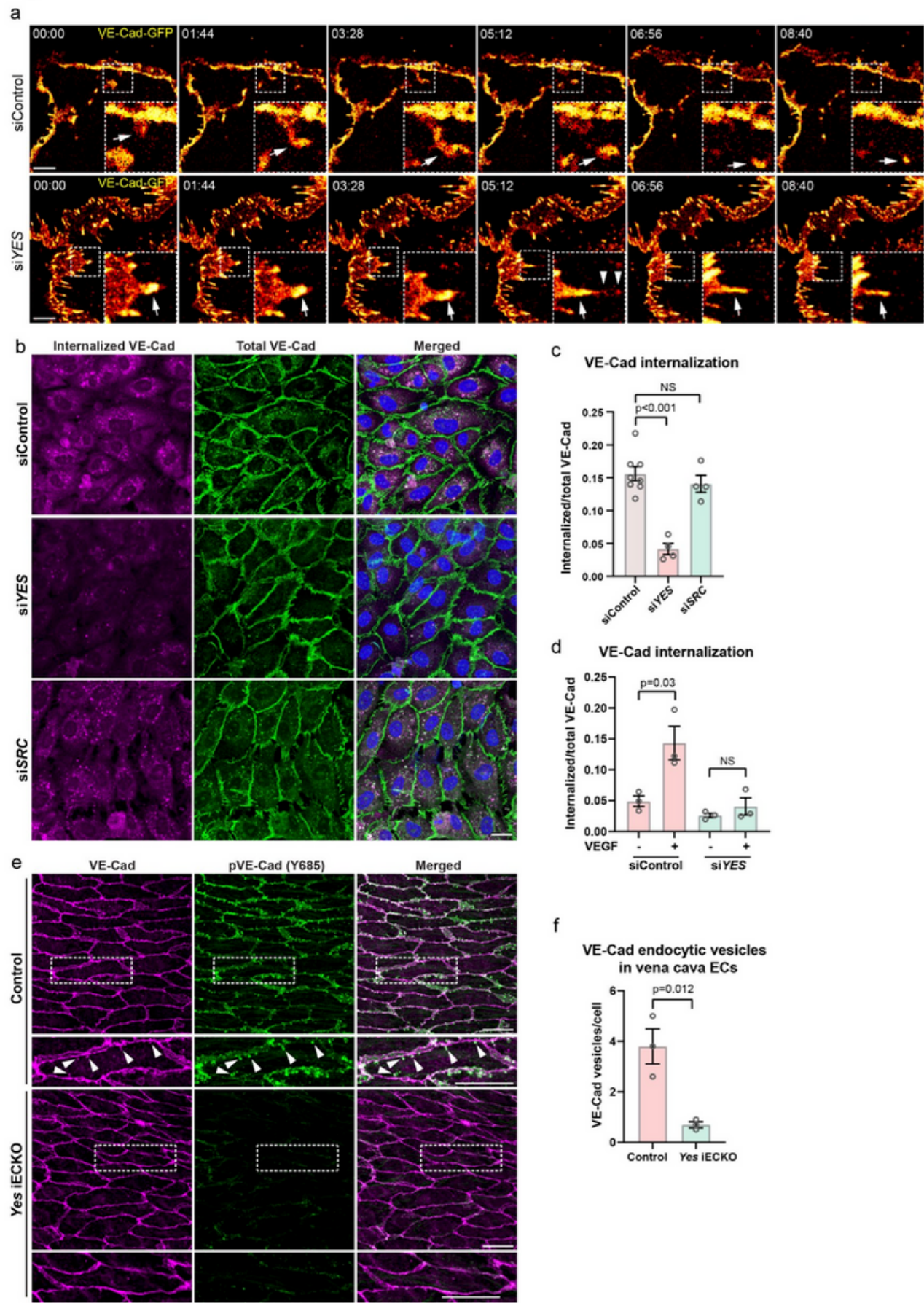

C VE-Cad internalization

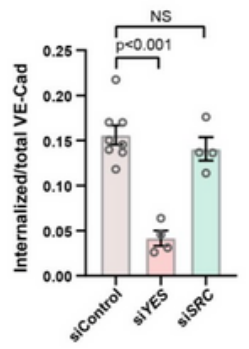

d VE-Cad internalization

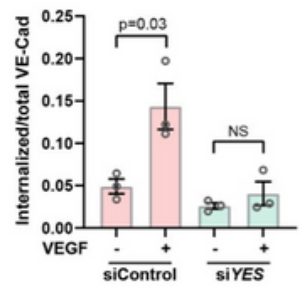

VE-Cad endocytic vesicles

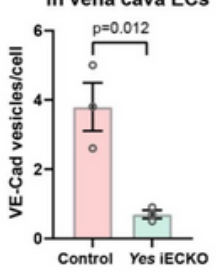

Figure 6

\section{YES regulates VE-cadherin dynamics}

(a) Static images from live imaging time series of HUVECs expressing GFP tagged VE-cadherin. Boxed areas are examples of different dynamics of VE-cadherin and are shown enlarged in the lower right. Arrows in the upper panel indicates an internalized VE-cadherin vesicle in control cells. Arrows in the 
lower panel indicates a VE-cadherin cluster at the junction of YES silenced cells. Arrowheads show detachment and internalization of VE-cadherin from the cluster at the junction. Timestamps are min:sec. Scale bars, $10 \mu \mathrm{m}$. (b) Antibody feeding assay showing internalized VE-cadherin (magenta) in control, si YES and siSRC HUVECs, co-stained with total VE-cadherin (green) and nuclei (blue). Scale bar, $20 \mu \mathrm{m}$. (c) Quantifications of internalized/total VE-cadherin in control, si YES and siSRCHUVECs. Control, $\mathrm{n}=8$ independent experiments; si YES, $\mathrm{n}=4$ independent experiments; si $S R C, \mathrm{n}=4$ independent experiments. (d) Quantification of internalized/total VE-cadherin in control and siYESHUVECs treated with VEGFA or not for 3 h. $n=3$ independent experiments. Representative images are shown in supplementary figure 7a. (e) Internalized VE-Cad vesicles at juxtamembrane regions in mouse vena cava ECs shown by immunostaining of VE-Cad (magenta) and pVE-Cad (Y685) (green). The cells highlighted in boxes are shown enlarged below. Arrowheads indicate internalized VE-cad vesicles. Scale bars, $20 \mu \mathrm{m}$. (f) Quantification of internalized VE-Cad vesicles/cell in control and Yes iECKO vena cava ECs. $\mathrm{n}=3$ mice for each group. Bar graphs show mean \pm S.E.M with individual data points. 
Figure 7
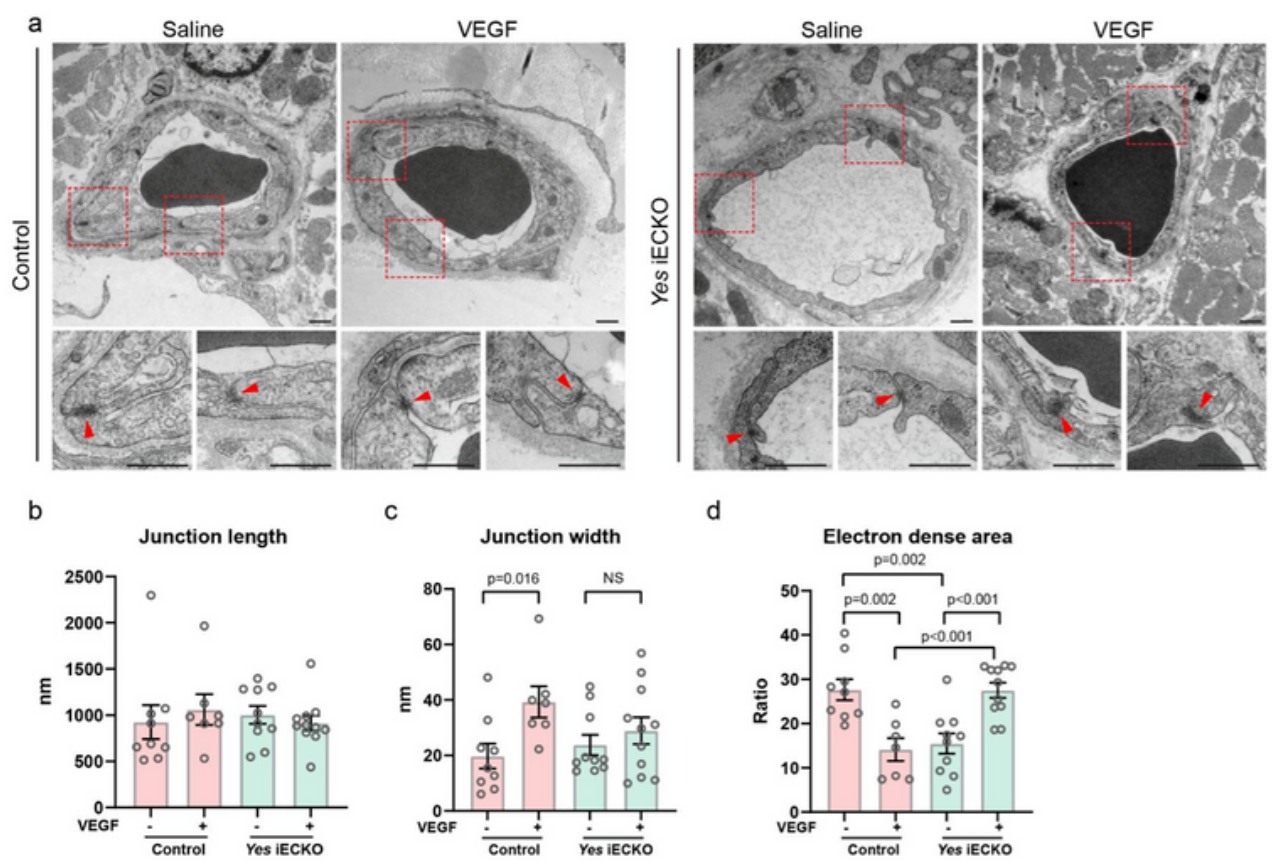

d
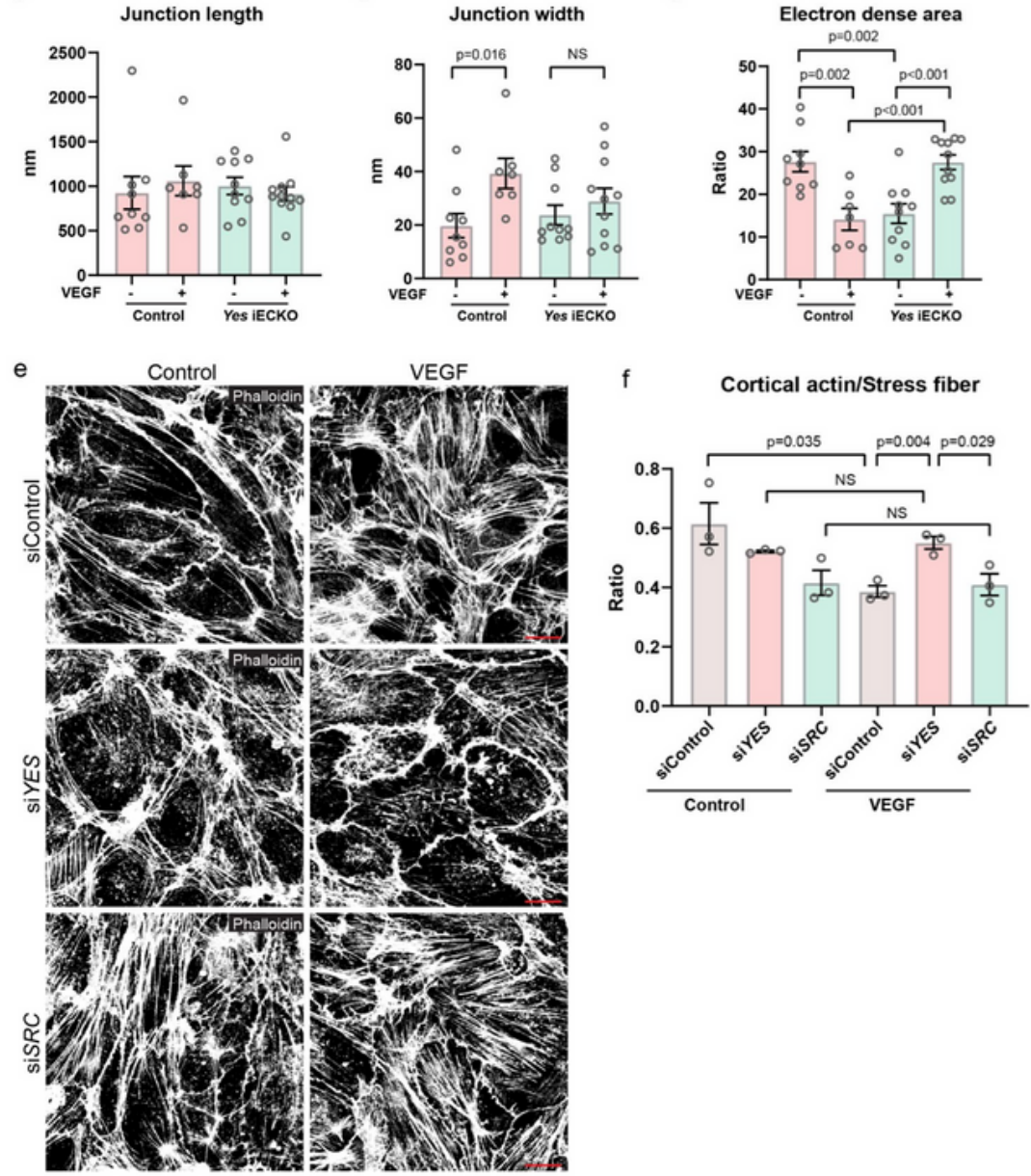

Figure 7

\section{Yes-deficiency impacts cell-cell junctions and actin organization}

(a) Endothelial junctions in mouse ear dermis vessels after treatment with saline or VEGFA164 visualized by transmission electron microscopy (TEM). Regions containing junctions are boxed and shown enlarged below. Arrowheads indicate electron dense area at the junctions. Scale bars, $500 \mathrm{~nm}$. Quantifications of 
junction length, junction width and ratios of electron dense area/junction length (b-d). Saline treated control mice, $n=9$; VEGFA treated control mice, $n=7$; saline treated Yes iECKO mice, $n=10$; VEGFA treated Yes iECKO mice, $n=11$. (e) Staining with phalloidin shows the arrangement of actin stress fibers and cortical actin in HUVECs treated with control, YES or SRC siRNA followed by VEGFA stimulation for 15 min. Scale bars, $20 \mu \mathrm{m}$. (f) Quantifications of the integrated intensity of actin stress fiber normalized to cell numbers in control, YES or SRC silenced HUVECs. $n=3$ independent experiments. Bar graphs show mean \pm S.E.M with individual data points. 
Figure 8
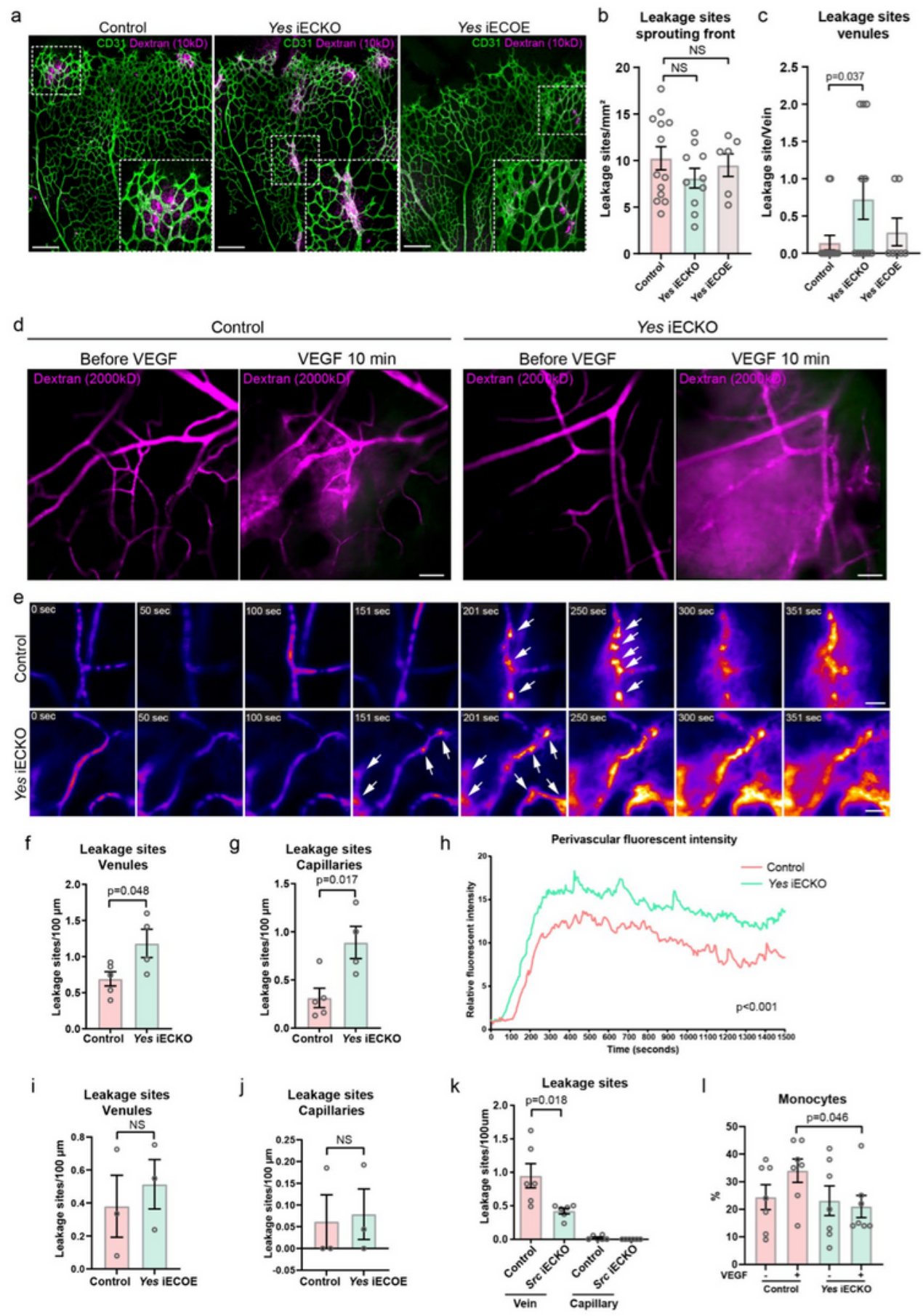

\section{Figure 8}

\section{YES is required for vascular integrity and leukocyte extravasation}

(a) Detection of leakage of $10 \mathrm{kD}$ dextran (magenta) in fixed P6 retinas of control, Yes iECKO and Yes iECOE mice. Vessels are shown by CD31 staining (green). Boxed areas are leakage sites, shown in enlarged pictures. Scale bars, $200 \mu \mathrm{m}$. (b) Quantification of leakages sites with perivascular dextran 
accumulation at the sprouting front. Control, $n=9$ retinas; Yes $i E C K O, n=10$ retinas; Yes $i E C O E, n=6$ retinas. (c) Quantification of leakages sites at retinal veins. Control, $n=14$ veins in 9 retinas; Yes $\mathrm{EECKO}, \mathrm{n}=11$ veins in 10 retinas; Yes $\mathrm{iECOE}, \mathrm{n}=7$ veins in 6 retinas. (d) Static images from intra-vital time series imaging on mouse ear dermal vessels before and 10 min after VEGFA injection. Leakage was visualized by the extravasation of circulating $2000 \mathrm{kDa}$ dextran (magenta) into the perivascular area. Scale bars, $50 \mu \mathrm{m}$. (e) Heat map images showing the appearance of individual leakage sites (arrows) after VEGFA injection. Scale bars, $20 \mu \mathrm{m}$. (f, $\mathbf{g})$ Quantification of individual leakage sites at veins and capillaries induced by VEGFA. Control, $n=5$ mice, Yes iECKO, $n=4$ mice. (h) VEGFA-induced changes in perivascular fluorescent intensity measured at 2 second intervals for 1500 seconds. Curves show mean values of 8 leakage sites analyzed from 5 control mice and 10 sites analyzed from 4 Yes iECKO mice. (i, j) Quantification of individual leakage sites at veins and capillaries induced by VEGFA. Control, n=3 mice, Yes iECOE, n=3 mice. (k) Quantification of individual leakage sites at veins and capillaries in ear dermis of control and Src iECKO mice after VEGFA injection. Control, $n=6$ mice, Src iECKO, $n=6$ mice. (I) FACS analysis of monocytes/macrophages (CD11b+, LY6G-) extravasation in peritoneal fluid from mice $24 \mathrm{~h}$ after i.p. injection with saline or VEGFA, $n=7$ mice for each group. Bar graphs show mean \pm S.E.M with individual data points.

\section{Supplementary Files}

This is a list of supplementary files associated with this preprint. Click to download.

- SupplementaryinformationJinetal211208merged.pdf

- Supplementaryvideo1.mp4

- Supplementaryvideo2.mp4

- Supplementaryvideo3.mp4

- Supplementaryvideo4.mp4

- Supplementaryvideo5.mp4

- Supplementaryvideo6.mp4

- Supplementaryvideo7.mp4

- Supplementaryvideo8.mp4

- Supplementaryvideo9.mp4

- Supplementaryvideo10.mp4

- Supplementaryvideo11.mp4 